\title{
Signatures of rapid synaptic learning in the hippocampus during novel experiences
}

James B. Priestley ${ }^{1,2,3, \dagger}$, John C. Bowler ${ }^{1,2}$, Sebi V. Rolotti ${ }^{1,2}$, Stefano Fusi $^{1,3,4}$, Attila Losonczy ${ }^{1,4, \dagger}$

1 Department of Neuroscience

2 Doctoral Program in Neurobiology and Behavior

3 Center for Theoretical Neuroscience

4 Mortimer B. Zuckerman Mind Brain Behavior Institute

Columbia University, New York, NY 10027 USA

$\dagger$ Correspondence should be addressed to:

J.B.P.: jbp2150@columbia.edu; A.L.: al2856@columbia.edu 


\section{Summary}

Neurons in the hippocampus exhibit striking selectivity for specific combinations of sensory 2 features, forming representations which are thought to subserve episodic memory. Even during a 3 completely novel experience, ensembles of hippocampal "place cells" are rapidly configured such 4 that the population sparsely encodes visited locations, stabilizing within minutes of the first 5 exposure to a new environment. What cellular mechanisms enable this fast encoding of experience? 6 Here we leverage virtual reality and large scale neural recordings to dissect the effects of novelty 7 and experience on the dynamics of place field formation. We show that the place fields of many 8 CA1 neurons transiently shift locations and modulate the amplitude of their activity immediately 9 after place field formation, consistent with rapid plasticity mechanisms driven by plateau potentials 10 and somatic burst spiking. These motifs were particularly enriched during initial exploration of a 11 novel context and decayed with experience. Our data suggest that novelty modulates the effective 12 learning rate in CA1, favoring burst-driven field formation to support fast synaptic updating during 13 new experience.

\section{Introduction}

Learning in neuronal systems is complicated by a fundamental tension between stability and plasticity (Carpenter \& Grossberg 1991). Networks with fast learning rates encode new information with high fidelity at the expense of overwriting older patterns, while a slow learning rate can preserve existing structure yet frustrate the encoding of novel information. Theoretical studies of neuronal memory capacity suggest that optimal solutions involve concerted processes operating on 20 a spectrum of timescales (Roxin \& Fusi 2013, Benna \& Fusi 2016). These ideas harmonize with 21 models of multi-stage memory systems in the brain (McClelland et al. 1995): fast-learning circuits 22 can quickly capture detailed memories of new episodes, which are progressively transferred and 23 integrated into slower systems downstream.

The mammalian hippocampus is intimately involved in the formation of episodic memories, and 25 likely mediates an intermediate stage of processing and storage of experiential information prior to 26 long-term storage in the cortex (McClelland et al. 1995). While generally viewed as a short-term 27 
memory system, hippocampal dynamics exhibit a diversity of time constants, both at the level of

its sub-networks (Mankin et al. 2015, Ziv et al. 2013) and cellular plasticity mechanisms (Bittner 29

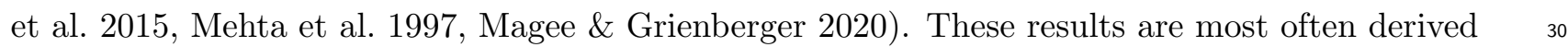
from the study of "place cells", excitatory neurons in the hippocampus that are active in specific 31 locations in an environment during exploration (Moser et al. 2008). Spatial behaviors provide a 32 convenient model for studying memory, as the various sensory settings that animals encounter in 33 the environment are organized into highly relational neural representations in the hippocampus 34 (Eichenbaum 2017). Novel population codes develop with remarkable speed, requiring only a few 35 exposures to an environment before a new set of place fields is learned that spans the available 36 space (Wilson \& McNaughton 1993, Frank et al. 2004).

A unique synaptic learning rule was recently discovered in the CA1 subregion of the hippocampus, by which pyramidal neurons formed stable place fields within just a few laps after 39 burst firing was recorded from the neuron at a particular location in the environment (Bittner et al. 40 2015, 2017). The sudden emergence of tuning in previously silent neurons marks a rapid reconfiguration of the weights of synapses that were active around the time of the burst event (Bittner et al. 2017, Milstein et al. 2020), with an asymmetric envelope that extends to inputs active several seconds before the event occurred. This "behavioral timescale synaptic plasticity" 44 (BTSP) is a notable departure from conventional plasticity schemes. Its expression depends on the 45 presence of somatic burst firing driven by plateau potentials, which reflect nonlinear input integration in the dendritic arbor of pyramidal cells (Epsztein et al. 2011 ). These events could be ${ }_{47}$ gated by the presence of other factors such as inhibition, neuromodulation or "instructive" inputs 48 signaling reinforcement or novelty (Gerstner et al. 2018, Milstein et al. 2020), which could enable 49 the circuit to rapidly construct new representations during salient experiences.

It remains unknown what connection this plasticity mechanism has to the reorganization of 51 hippocampal responses that occurs when animals are exposed to different environments ("global 52 remapping", Muller \& Kubie (1987)) or in response to salient cues or reinforcement (Hollup et al. 53 2001, Zaremba et al. 2017, Dupret et al. 2010). The presence of plateau potentials or associated 54 somatic burst spiking reliably leads to the formation of place fields de novo (Bittner et al. 2015, 55 Diamantaki et al. 2018), but other experiments have reported that new place fields can appear in 56 
the absence of these signatures both in familiar and novel environments (Cohen et al. 2017$)$. 57 Clearly the network's synaptic matrix does not start from a blank slate when learning from each 58 new episode; prior experience in other environments may already provide a weight distribution that 59 produces location-specific, suprathreshold spiking for some neurons, or subthreshold tuning that 60 could be amplified to unmask new receptive fields through other plasticity mechanisms (Lee et al. 61 2012, McKenzie et al. 2021). We lack clarity on the extent to which BTSP contributes to learning 62 hippocampal representations in these different scenarios, which could provide insight on how the ${ }_{63}$ network learning rate may change in response to factors such as novelty or salience.

In this work, we conducted a large scale, longitudinal analysis of place field formation during 65 familiar and novel experiences in order to search for signatures of BTSP and how they may change $\quad 66$ as a function of experience. Using 2-photon functional calcium imaging, we surveyed thousands of ${ }_{67}$ place fields and identified an enrichment of BTSP-like dynamics during the initial exposures to a 68 new environment, which then decayed over the course of several days. Our findings suggest that ${ }^{69}$ BTSP is a widespread phenomenon in CA1, and illustrate an experience-dependent regulation of 70 plasticity that could be controlled by internal or external factors to dynamically tune the learning 71 rate of hippocampal representations.

\section{Results}

We constructed a virtual reality system for head-restrained mice, comprising 5 LCD displays 74 surrounding a running wheel; movement through the virtual environments was yoked to a rotary 75 encoder on the wheel axle. We combined this apparatus with 2-photon functional calcium imaging 76 in order to record CA1 neural populations as mice explored the virtual contexts (Fig 1A, S1, see 77 Methods for details). Mice were stereotactically injected with an rAAV vector encoding the calcium 78 sensor GCaMP6f under the control of the synapsin promoter, targeted to the CA1 pyramidal layer. 79 We then implanted a chronic window above the hippocampus to provide optical access for imaging 80 experiments (Lovett-Barron et al. 2014). All imaging data was post-processed in Suite2p ${ }_{81}$ (Pachitariu et al. 2017) for motion correction, cell detection, and extraction of raw fluorescence 82 traces (Fig 1B). Signals were neuropil-corrected and detrended for baseline drift, and putative 83 events were detected using the OASIS spike inference algorithm (Friedrich et al. 2017). Imaging 84 
A

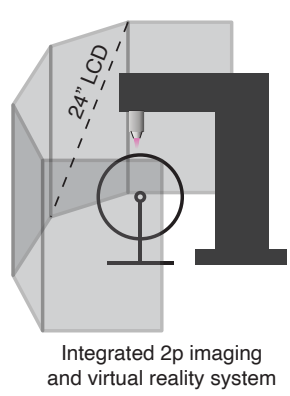

B

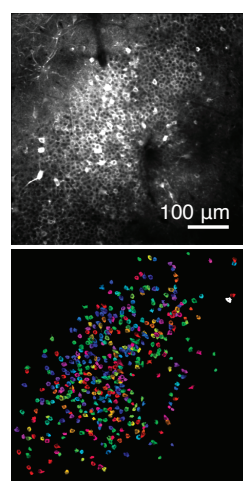

C

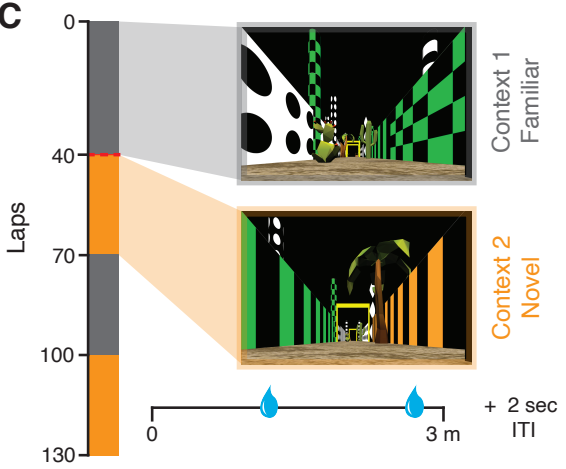

D

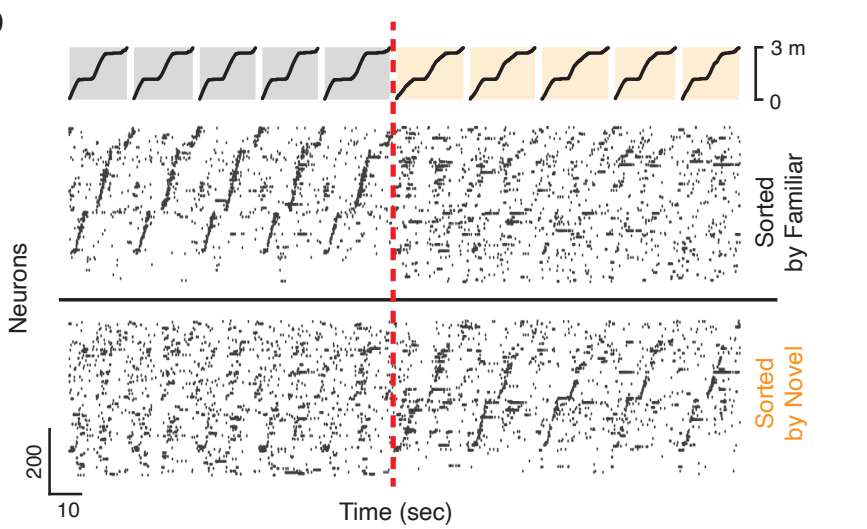

E

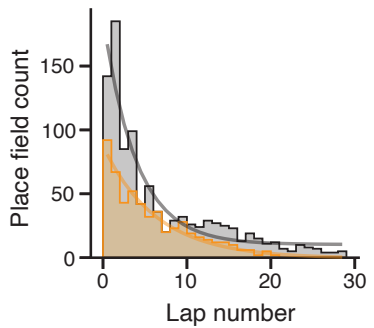

Figure 1. Formation of CA1 representations in novel virtual environments. A: Schematic of integrated 2-photon microscope and virtual reality system, for simultaneous in vivo functional calcium imaging and head-fixed behavior experiments. B: Top: example 2-photon field-of-view (FOV) of CA1 pyramidal neurons expressing the fluorescent calcium reporter GCaMP6f. Bottom: spatial masks of functionally identified ROIs from the same FOV. C: Structure of the task. Each session consisted of 130 laps, alternating in blocks between a familiar (grey) or novel (orange) context. Both environments were $3 \mathrm{~m}$ in length. Mice were trained to run for sucrose rewards that were delivered at two fixed locations on each lap $(\sim 1.2$ and $2.7 \mathrm{~m})$; the relative position of reward was the same in both contexts. The experiment was repeated for 3 consecutive days using the same familiar/novel contexts. D: Ensemble neural activity from an example Day 1 session, shown for \pm 5 laps around the very first exposure to the novel context (transition marked in red). Neurons are sorted by their peak firing location on the track, calculated from their average spatial tuning in either familiar (top) or novel (bottom) context laps. A new sequence of place fields rapidly organizes within the first few trials in the novel context. E: Place field accumulation in the first trial blocks of the familiar and novel contexts on Day 1, shown as a histogram of the formation laps (the first lap that a cell fired within its place field).

data was collected from approximately the same area of CA1 for each 3-day experiment sequence, 85 although we did not attempt to track the identity of individual neurons across sessions. The resulting dataset consisted of an average of $391 \pm 39$ CA1 neurons per session $(35$ sessions from 6 mice).

We first trained mice to run for sucrose rewards in a 3 meter virtual environment. All virtual environments began and ended in a darkened tunnel; entering the exit tunnel triggered a 2 sec inter-trial interval (ITI) during which the screens would remain dark, and after which the animal 
over 100 laps in under an hour. To study the dynamics of place field formation during novel experience, we leveraged the virtual reality system to rapidly alternate between familiar and novel environments multiple times during a single experiment (Figure $1 \mathrm{C}$ ). In each session, mice ran laps switches were uncued; the mouse was simply teleported to the other environment at the end of the last ITI of a given block. We repeated the context alternation experiment across 3 consecutive days using the same familiar/novel contexts, to examine the effects of increasing familiarity on CA1 contexts (the familiar context remained the same).

\ Consistent with previous work using head-fixed VR preparations (Sheffield et al. 2017, Zhao responses on each lap (Fig 1D). Examining the very first exposure to the novel context on Day 1, a 104 sequence of place fields was rapidly configured in the first few laps following the context switch; the 105 majority of place fields in the novel environment appeared in these early laps, with field 106 accumulation decaying roughly exponentially with experience (Fig 1 E) similar to prior reports 107 (Sheffield et al. 2017). Gross place field accumulation in the familiar context was two-fold greater in 108 the initial laps and decayed faster compared to the novel context $(\tau=3.9$ in Familiar and $\tau=6.4 \quad 109$ in Novel for exponential decay fit to Fig 1 $\mathrm{E}$ ). These features agree with our intuition that many 110 place fields in the familiar environment are due to prior learning (and so they immediately appear 111 within the first laps in the session), while the novel representation may continue to grow through 112 ongoing plasticity over a longer time period.

We analyzed the dynamics of place coding across laps in each context block in order to identify 114 activity signatures that may be consistent with different underlying plasticity mechanisms. One 115 hallmark of plateau-induced field formation in CA1 is the backward shift of spatial tuning, relative ${ }_{116}$ to the location of burst firing during the formation lap (i.e. the lap when the cell first fires near its ${ }_{117}$ place field). This transient shift is a consequence of the asymmetric plasticity kernel of BTSP 118 (Bittner et al. 2017). If new place fields in the novel context form predominantly through BTSP, 119 then this should induce a transient backward drift that is measurable in the population tuning of $\quad 120$ space during the first few laps in the environment. Since this is the time period during which the ${ }_{121}$ 
majority of place fields form (Fig $1 \mathrm{E}$ ), the population drift could arise from the cumulative effect of ${ }_{122}$ many fields shifting on the same lap, an effect that can notably be measured without first ${ }_{223}$ identifying specific place fields or their formation lap.

To quantify any spatial shift between population representations on different trials, we estimated 125 the population spatial cross-correlation between pairs of trials (Figure 2A) by repeatedly computing 126 a population vector correlation as we systematically displaced one trial's neural responses in space ${ }_{127}$ across a range of spatial lags $( \pm 75 \mathrm{~cm})$. For the example trial pair in Figure 2A, the peak ${ }_{128}$ correlation occurs at a spatial lag of $+6.6 \mathrm{~cm}$, indicating that the activity in trial $a$ appears forward-shifted in space relative to trial $b$. In Figure $2 \mathrm{~B}$, we summarized the resulting spatial shifts 130 between all trial pairs as a matrix, shown for Day 1 experiments (when the animal is exposed to the 131 novel context for the very first time) separately for each context trial block. Since the sign of the 132 the spatial shift between two trials is reversed when the order of comparison is reversed (i.e. trial $a \quad 133$ is after trial $b$, so $b$ is before $a$ ), these matrices are antisymmetric. In general, the upper triangle of 134 the shift matrices was slightly positive, indicating that the population tuning tended to drift 135 backward in space relative to earlier laps in each block (and conversely, the slightly negative lower ${ }_{136}$ triangle reflected the tendency of earlier laps to be shifted forward in space relative to later laps). $\quad{ }_{137}$ However, the shift pattern during the first exposure to the novel context (Switch 1) was markedly 138 different from other trial blocks: early trials in the context showed a far more exaggerated shift 139 forward in space relative to later trials. We summarized this trend by computing the average 140 pairwise shift for each trial per block (Figures 2 $\mathrm{C}, \mathrm{S2}$ ), which showed a large but transient forward 141 shift of population spatial tuning during the early trials of the first block in the novel context. This 142 shift decayed rapidly within the first 10 trials in the new context, notably overlapping with the ${ }^{143}$ period during which the majority of new place fields appeared in the novel context (Figure 1 $\mathrm{E}$ ). $\quad{ }_{144}$

The representation in the novel context consistently showed a transient, backward drift during $\quad 145$ the first exposure across mice on Day 1 (Figures 2D, S3), but this effect was highly experience ${ }_{146}$ dependent. We repeated the context switch protocol over a 3 day period, and found that the ${ }_{147}$ greatest drift was reliably observed during Day 1, when the animals were exposed to the novel 148 context for the very first time (Figures 2D, E). It is possible that this transient drift is due to the ${ }_{149}$ rapid acquisition of new place fields during the initial laps in the novel context. If many neurons 
A
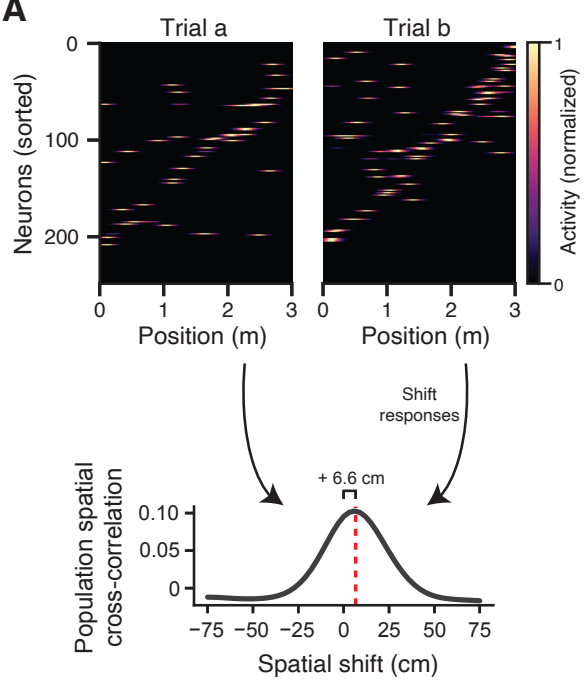

D

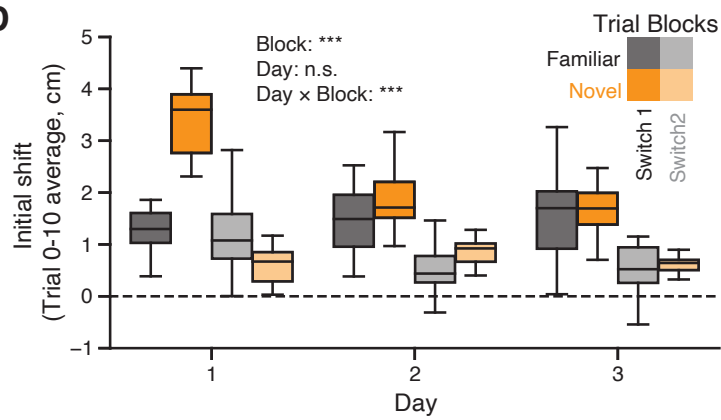

B

C
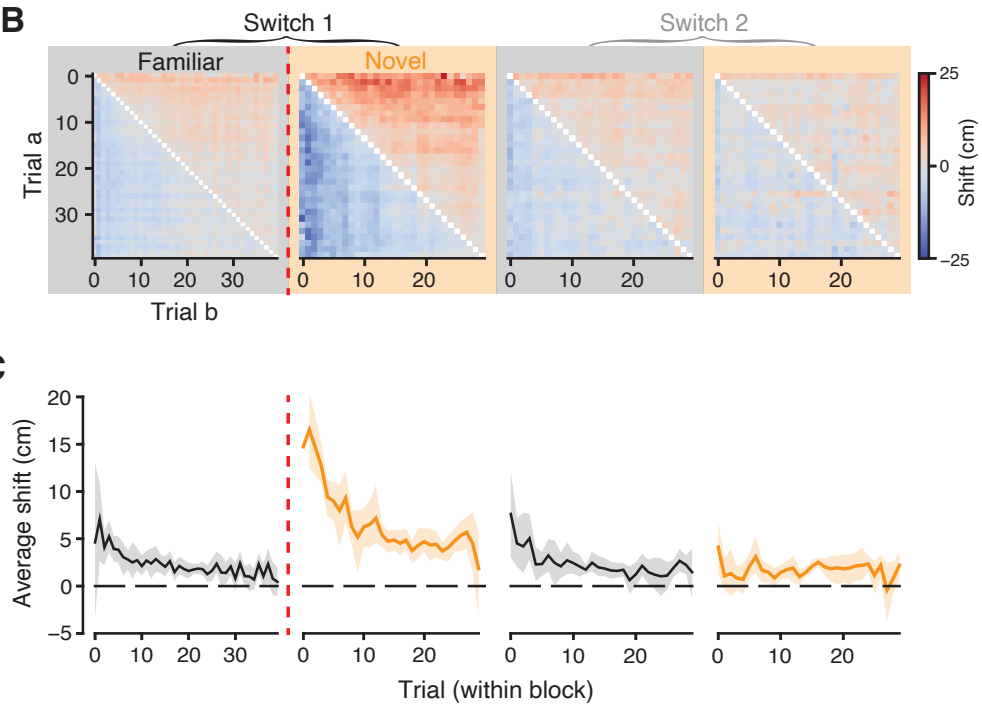

$\mathbf{E}$

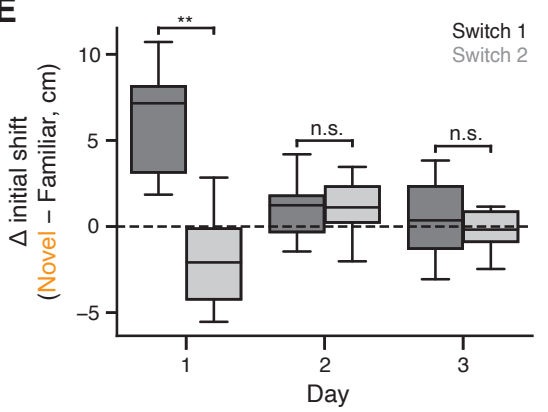

Figure 2. Transient, experience-dependent drift of CA1 ensemble coding. A: Top: spatial tuning of the neural population during two example trials in the same context. Neurons are sorted by the location of their peak firing across all laps in the context. Bottom: population spatial-cross correlation computed between the two trials. The correlation at different spatial lags is obtained by shifting each neuron's tuning in trial $b$ before forming the population vector. Red dashed line indicates the center of mass (COM) of the spatial cross-correlation curve. A shift at positive lags indicates the responses in trial $a$ are at positions ahead of the responses in trial $b$. B: Spatial shifts (COM) between all pairs of trials during each trial block on Day 0 (average of $n=11$ experiments). C: Average shift for each trial in the experiment, mean and 95\% confidence interval across Day 0 experiments. See Figure S2 for individual mice data across all days. D: Average representation shifts in the first 10 trials of each block, summarized over all 3 days of the experiment; linear mixed-effects model with main effects of trial block and day (significance shown in inset). E: Difference in initial representation drift between the novel vs familiar contexts, for each context switch and day; Wilcoxon signed-rank test. ${ }^{*} p<0.05,{ }^{* *} p<0.01,{ }^{* * *} p<0.001$ 
formation in the first exposure to the novel context.

We sought to connect this population-level observation with the behavior of individual place fields (Figure 3A). Due to the asymmetric plasticity kernel of BTSP, individual place fields should acutely shift backward (relative to the direction of animals' motion) between the formation lap and 161 subsequent laps. We detected place fields in each context block separately for each experiment, and 162 measured how displaced spatial activity on the formation lap was from activity on remaining laps ${ }_{163}$ (Figures 3B, C). Note that we use "formation lap" to denote the lap where we first detected stable 164 firing within a place field in a given trial block, but this is not meant to imply that all fields form 165 through ongoing plasticity (i.e. many likely appear simply due to prior learning). Comparing 166 formation lap shifts across different trial blocks on Day 1, we found a clear increase in the displacement of the formation lap's tuning curve specifically during the first exposure to the novel 168 context, relative to the other trial blocks (Figure 3D). Similar to the population drift, the greatest 169 field shifts were observed during Day 1 on the first context switch (Figure $3 \mathrm{E}$ ). Examining 170 lap-by-lap displacements of place field activity, we also found that the shifting was most pronounced on the formation lap, and that place fields did not generally continue to drift after the 172 first few trials following place field formation (Figure S4). These results align with the 173 experience-dependent drift in population tuning described in Figure 2, suggesting that the latter 174 arises due to the cumulative effect of many individual cells undergoing acute tuning shifts as they 175 formed their place fields.

The long timescale of BTSP also induces a correlation between the width of place fields and the 177 speed of the animal during the plasticity event: if the animal runs faster, the potentiated inputs 178 will span a larger region on the track (Bittner et al. 2017). For every identified place field, we 179 measured the velocity of the animal as it traversed the place field on the lap of field formation. If ${ }_{180}$ many fields during a trial block form through BTSP, then many of these first passes through the ${ }_{181}$ place field could contain plateau-driven burst spiking that leads to field formation. Examining the 182 joint distribution of formation lap velocities and the width of the associated place fields, we found ${ }_{183}$ that these variables were correlated in both the familiar and novel context (Figure $3 \mathrm{~F}$, data shown 184 for Day 1, switch 1). However, this correlation was stronger in the novel context, and the linear fit 185 produced a significantly greater slope compared to the familiar context (Figure $3 \mathrm{G}$ ). The difference ${ }_{186}$ 
bioRxiv preprint doi: https://doi.org/10.1101/2021.07.02.450956; this version posted July 3, 2021. The copyright holder for this preprint (which was not certified by peer review) is the author/funder, who has granted bioRxiv a license to display the preprint in perpetuity. It is made available under aCC-BY-NC-ND 4.0 International license.

between novel and familiar slopes was also experience-dependent; the first context switch on Day $1{ }^{187}$ exhibited the most significant slope difference by far across the entire experiment sequence. These 188 results are again compatible with a greater fraction of place cells forming via BTSP during the first 189 exposure to the novel context.

A
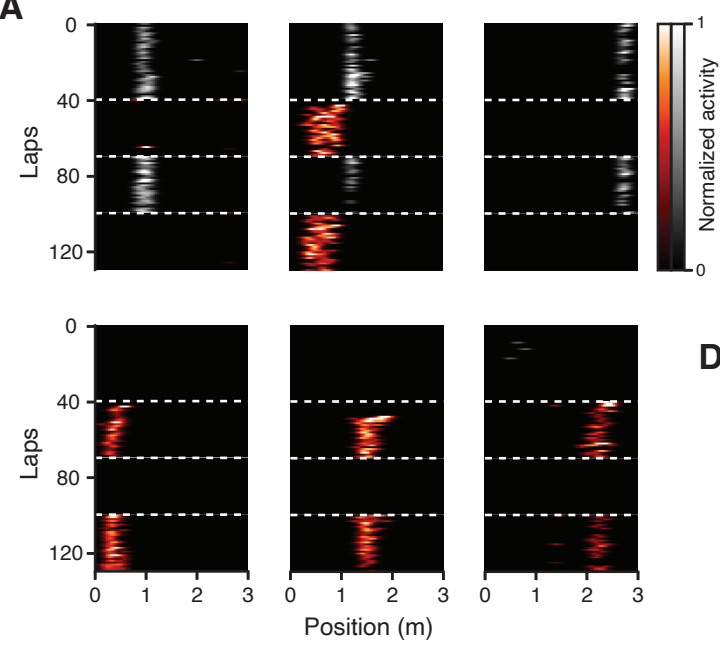

F

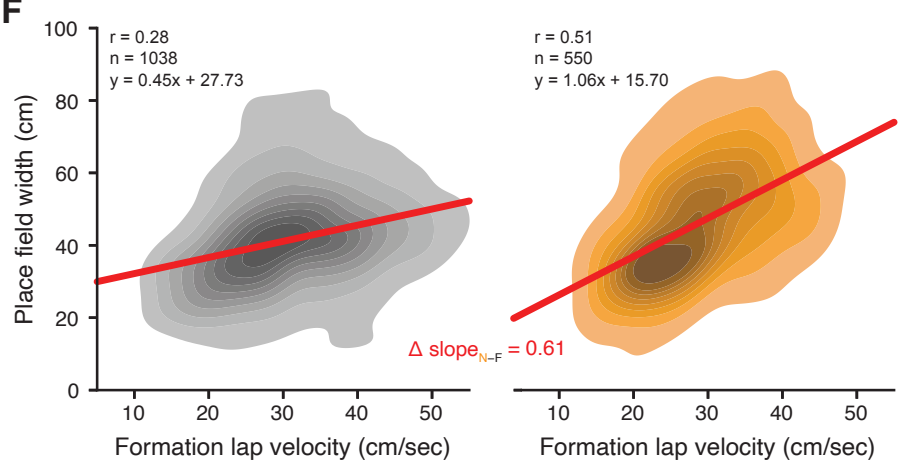

B

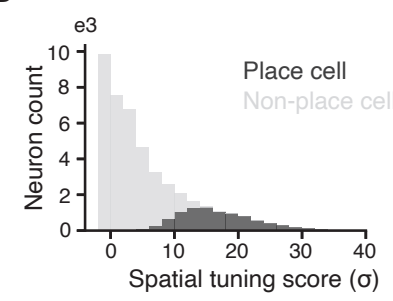

C

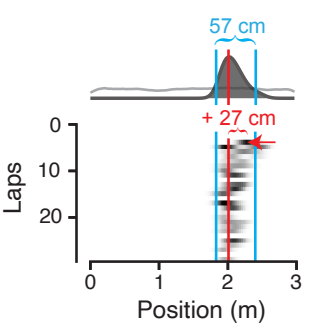

D

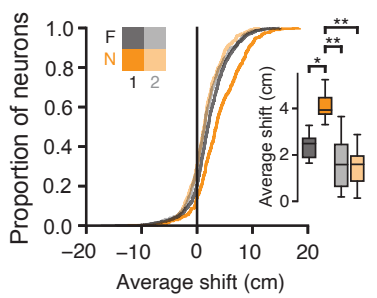

E

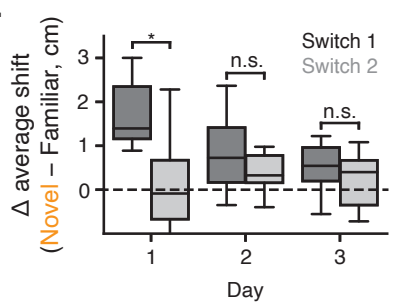

G

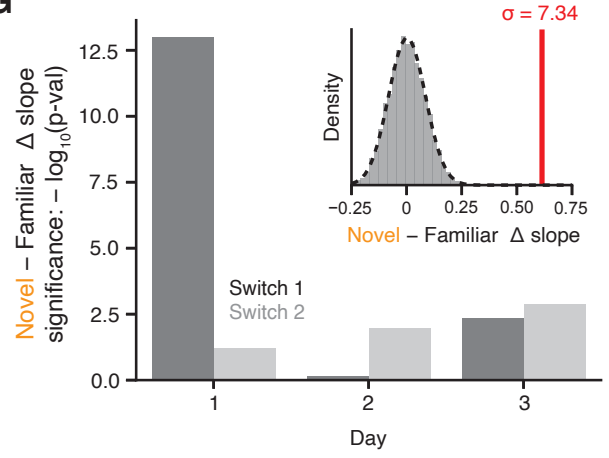

Figure 3. Place field dynamics are consistent with BTSP-mediated field formation in the novel context. A: Example simultaneously recorded CA1 neurons with place fields during Day 1 of the experiment. Stable place fields appeared in both the familiar and novel contexts. B: Distribution of spatial tuning scores for place and non-place cells. C: Characterizations of place field formation for an example neuron: field detection, field width, and formation lap shift. D: Distribution of place field shifts (distance between formation lap activity and the remaining in-field activity) during Day 1 of the experiment. Place fields in the first exposure to the novel context exhibit exaggerated spatial shifts on their formation lap. Inset: session-averaged place field shifts on Day 1 ( $\mathrm{n}=11$, Wilcoxon signed-rank test). E: Difference in place field shifts between Novel and Familiar contexts, for each context switch and day. The greatest change is seen during the first context switch on Day $1(\mathrm{n}=11$; linear mixed effects model gives significant main effects of Switch $\left({ }^{*}\right)$ and Day $(*)$, and Switch $\times$ Day interaction $(*)$; inset: Wilcoxon signed-rank test). F: Distribution of place field widths and the velocity of the animal as it traversed the place field during the formation lap on Day 1, switch 1. The linear fit is shown in red. The correlation between velocity and field-width is stronger in the novel context. G: Significance of the difference in regression slopes between Familiar and Novel as shown in F, for all days and switches. The $\Delta$ slope was compared to a null distribution created by randomly permuting the context labels of place fields for each context switch before recomputing the within-context regressions and between-context $\Delta$ slope (depicted in the inset for the Day 1, switch 1 results show in $\mathbf{F}$ ). Plotted is the negative $\log _{10} \mathrm{p}$-value derived from a Gaussian fitted to the null distribution. Positive values indicate a more significant difference. The strongest difference is seen for Day 1 , switch $1 .{ }^{*} p<0.05,{ }^{* *} p<0.01,{ }^{* * *} p<0.001$ 
We have so far considered population-level measures that correlate with the relative presence of $\quad 191$ BTSP-mediated place field formation between experimental conditions. Ideally we would like to 192 segregate individual place fields according to their pattern of spatial drift over laps. By examining 193 groups of place fields that share common spatiotemporal patterns of activity, we could potentially 194 identify a subgroup of place fields exhibiting BTSP-like characteristics or other dynamics, and study 195 additional properties of these classes and how their frequency changes with experience. Toward this 196 aim, we first took an unsupervised approach to identify a set of spatial activity patterns across laps 197 that were shared between place fields (Figures $4 \mathrm{~A}-\mathrm{C}$ ). For every place field in the dataset, we 198 examined the first 10 laps of spatial activity, starting from the formation lap. On each of these laps, 199 $150 \mathrm{~cm}$ of the spatial tuning curve was extracted, centered around the place field (Figure $4 \mathrm{~A}$ ). We 200 then concatenated all laps from the truncated spatial tuning matrices into a vector for every place 201 field. Consider the example place fields in Figure 4A: since we have centered each field's activity ${ }_{202}$ relative to its field center, the two "flattened" place fields appear as a relatively well-aligned series 203 of bumps. But notably, there is a large deviation in the location of firing during the formation lap: 204 Field 2 exhibits forward shifted activity relative to its place field center, whereas Field 1 remains ${ }_{205}$ centered. We hypothesize that many place fields will exhibit similar or other stereotyped patterns 206 of spatial shifts on certain trials. The core idea of the subsequent analysis is to employ 207 dimensionality reduction on these vectorial representations of the place fields, in order to identify a 208 small set of prototypical patterns that summarize the different trends of spatiotemporal activity. 209

All place fields in the dataset were gathered into a matrix $\mathbf{X}$, where each row is the ${ }_{210}$ spatiotemporal pattern of a single field during the first 10 trials from its formation lap (Figure 4B). 211 Each row was normalized by its mean to encourage the model to focus on shared variability between ${ }_{212}$ place fields. We sought a matrix decomposition $\mathbf{X}=\mathbf{W H}$, where each row of $\mathbf{H}$ would describe a ${ }_{213}$ pattern of spatial activity over laps, and each column of $\mathbf{W}$ would describe how that pattern ${ }^{214}$ contributed to individual place fields. There are many techniques for identifying a low-dimensional 215 set of shared patterns in this manner, but here we use non-negative matrix factorization (NMF), 216 since its strict non-negativity aids in interpreting the extracted components (activity described by 217 different components cannot be "cancelled out" due to negative values). In this setting, it is 218 straightforward to interpret the rows of $\mathbf{H}$ as a pattern of spatial activity over trials, where each ${ }_{219}$ 
A
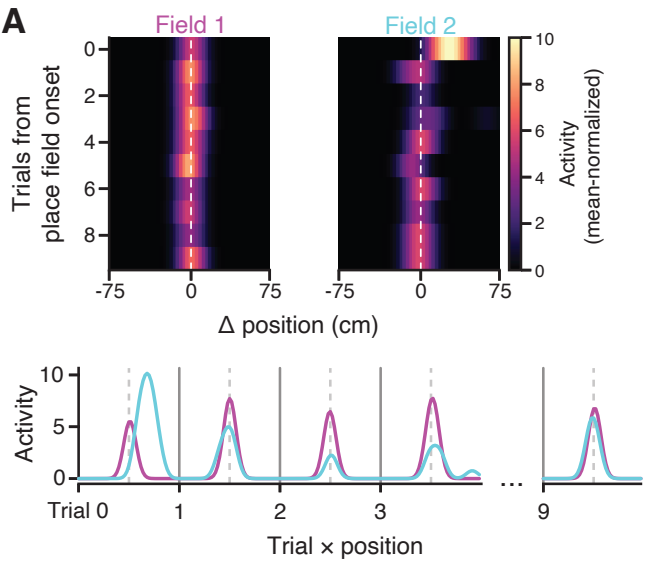

B

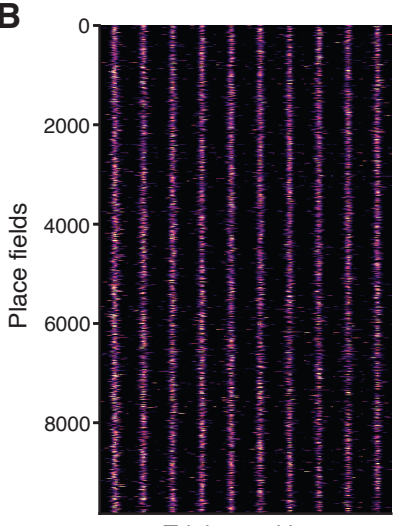

Trial $\times$ position

$E$

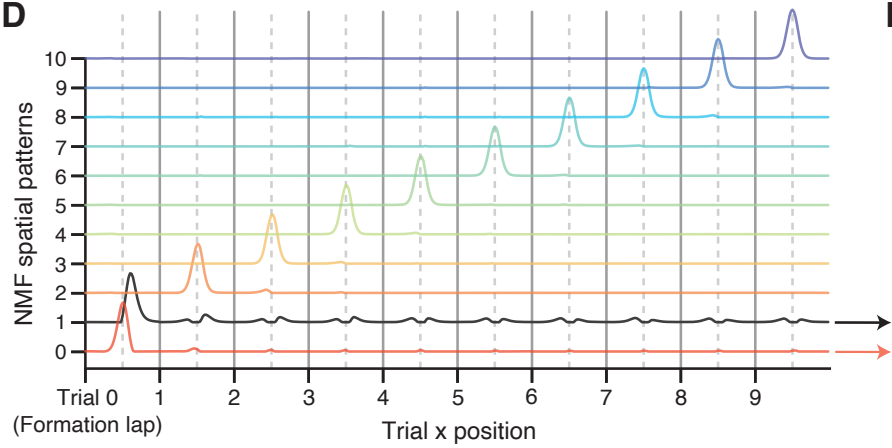

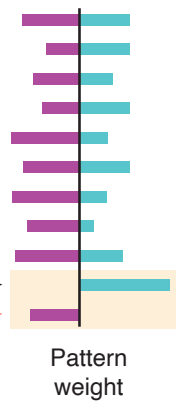

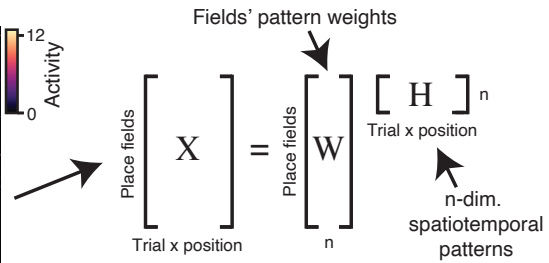

C

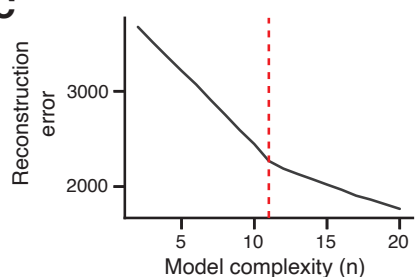

$\mathbf{F}$

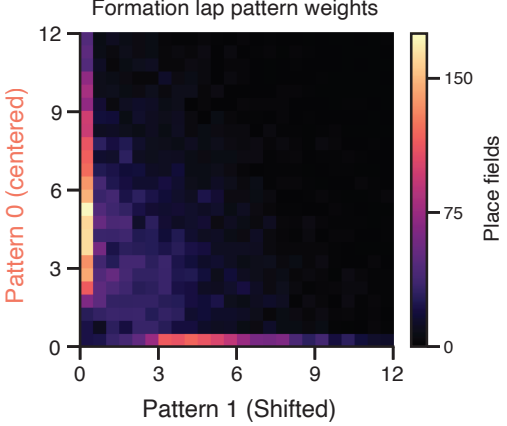

Figure 4. Factorizing place field responses across trials reveals distinct dynamics during the lap of place field appearance. A: Top: two example place fields, with data shown in a $150 \mathrm{~cm}$ window aligned to the place field center and truncated to the first 10 laps from the onset of place field formation. Bottom: the same fields, "flattened" so that each trial's spatial tuning curve is sequentially concatenated. B: Left: all identified place fields in the dataset with at least 10 laps of activity from onset, cropped and aligned as in A. Right: schematic of non-negative matrix factorization (NMF). Each place field's activity over trials is modeled as a weighted sum (W) of a small set of shared spatiotemporal patterns, H. C: Reconstruction error of $\mathbf{X}$ for different choices of the number of shared patterns $n$ in $\mathbf{H}$. There is a clear "elbow" at $n=11$, where model improvement slows. In Fig S6, we also show that this choice of $n$ gives the most interpretable patterns. D: Spatiotemporal patterns learned by NMF in the $n=11$ model. The model learns a separate pattern for each lap that produces the centered place field on that lap, plus an additional pattern (in black) on the formation lap that is shifted forward in space relative to the place field center. E: Weights of the spatiotemporal patterns in $\mathbf{D}$ for the example place fields shown in A. Trial-to-trial fluctuations in place field amplitude are captured by modulating the weight of the corresponding spatiotemporal pattern. Note the weight differences for the two "formation lap" patterns (yellow shading), which reflect the forwarded shifted activity for the cyan field. F: Joint distribution of pattern weights across all place fields for the two "formation lap" components. Field weights for the two patterns are uncorrelated.

individual place field in $\mathbf{X}$ is modeled as a weighted sum of those different patterns, with the

weights given by the rows of $\mathbf{W}$. As with any dimensionality reduction, it is necessary to choose the 
Strikingly, the 11-component model results in a very clean and interpretable partitioning of 226 variance in the place field dataset (Figure $4 \mathrm{D}$ ). 10 of the 11 components encoded the presence of a 227 centered place field on each of the 10 trials included in the data, while the 11th component encoded ${ }_{228}$ a forward-shifted place field on the first lap (i.e. the lap of field formation). This is a sensible way ${ }^{229}$ to decompose place fields: any individual field can now be reconstructed by appropriately weighting 230 each per-lap component according to the place field's amplitude on that lap, and the shifted 231 formation lap component can be used to account for variability due to BTSP-like field formation. 232 For example, consider the place fields in Figure 44. The pattern weights learned from the model ${ }_{233}$ accurately reflect the shift in formation lap activity for Field 2, and its reduced amplitude on later 234 laps relative to Field 1 (Figure $4 \mathrm{E}$ ). Notably, it is not immediately obvious that NMF should find 235 this per-lap representation; the model could instead identify components that are active on several ${ }_{236}$ or all laps, representing longer trends of place field amplitude and shifts that are shared between ${ }^{237}$ many neurons. Instead, our model's representation suggests that the variability in place field 238 activity in this dataset is best captured independently lap-by-lap for each place field.

Since the 11-component model converged on this orderly per-lap solution, we wondered if a 240 10-component model is sufficient to partition the data similarly (since 10 laps are included in $\mathbf{X}$ ), 241 but were surprised to find that it failed to do so. Instead, the 10-component model still prioritized 242 modeling the additional formation lap variance using two components (centered and shifted 243 versions), which resulted in another component encoding a mixture of multiple later trials (Figure 244 S6B). We studied the components learned by models of varying complexity, and found that lower 245 dimensional models invariably resulted in components that encoded mixtures of multiple laps, while 246 adding additional components beyond 11 simply split later lap's place fields into two smaller fields, 247 symmetrical about the place field center, while still enriching the formation lap representation with 248 additional forward-shifted patterns (Figure S6). Overall, the 11-component model resulted in the ${ }_{249}$ most interpretable components, and in light of the clear inflexion point in the loss function (Figure 250 4C), we focused our remaining analysis on this decomposition.

The representation of formation lap activity in the model illustrates the additional variability 252 present during the lap of place field formation across the dataset. Inspecting the joint distribution 253 of fields' weights on these two components, we found that they contributed to mostly orthogonal 254 
groups of place fields (Figure $4 \mathrm{~F}$, note the concentration about the axes). In fact, the two formation 255 lap components had the most orthogonal weight vectors out of any pair in the model (Figure S7A). 256 The shifted formation lap component was also weakly anticorrelated with all remaining lap

A

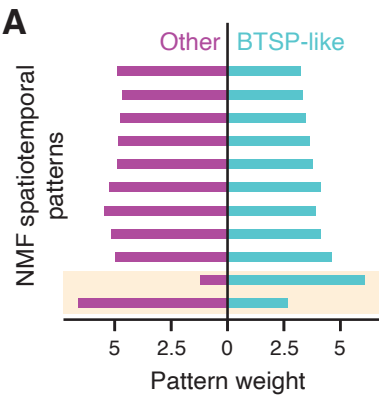

D

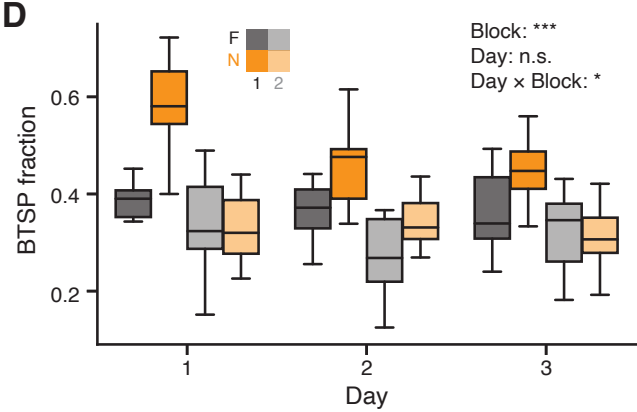

B

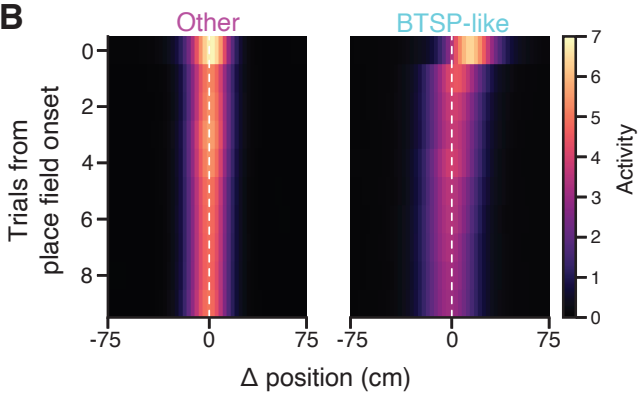

E

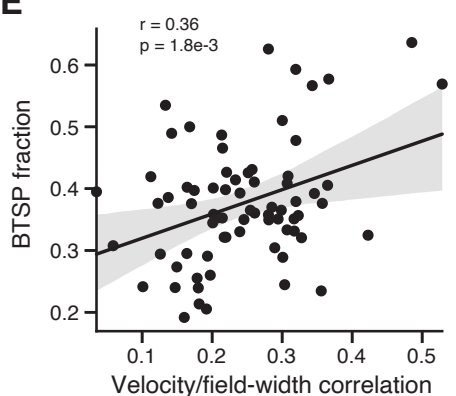

C

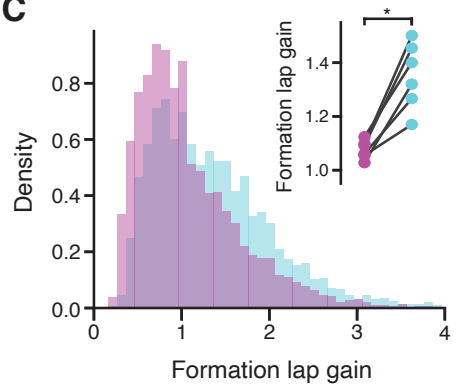

$\mathbf{F}$

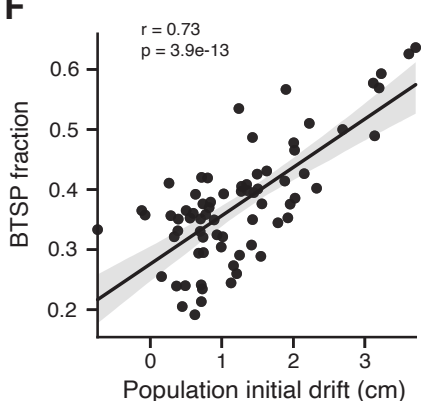

Figure 5. BTSP-like field formation is enriched in the novel context and decays with experience. A: Place fields were divided into two groups via K-means clustering in NMF space (i.e., clustering the rows of $\mathbf{W}$, see Methods and Figure S7). Plotted is the average NMF pattern weights for place fields in the two groups, labeled as BTSP-like and other. BTSP-like fields have far greater weight on the shifted, plateau-like component on the formation lap, and generally less weight on later lap components compared to the other cluster. Components are sorted as in Figure 4D,E. B: Average spatial tuning over trials for place fields in each group. As suggested by the pattern weights in A, the BTSP-like group exhibits forward shifted activity on the formation lap, and the formation lap amplitude is greater than later trials. C: Formation lap activity gain for each place field, calculated as the peak activity on the formation lap divided by the peak activity from the average of the remaining laps. BTSP-like cells show higher formation lap gain (Kolmogorov-Smirnov test, $p<6.68 \times 10^{-41}$ ). Inset: average gain for place fields in each group, for each mouse (Wilcoxon signed-rank test). D: Fraction of place cells classified as BTSP-like in each experiment, shown separately for each trial block on each day. BTSP-like place field formation is enriched during the first exposure to the novel context, and decays with experience. (linear mixed effect model with main effects of trial block and day, significance inset). E: Correlation between BTSP fractions and the slope of the velocity/field-width regression. F: Correlation between BTSP fraction and the population-level initial drift score. In $\mathbf{E}$ and $\mathbf{F}$, each point is a trial block from a single day and single mouse. ${ }^{*} p<0.05,{ }^{* *} p<0.01,{ }^{* * *} p<0.001$ 
$5 \mathrm{~A}$, we plotted the cluster centers for the two groups in the NMF component space. The two

How do these two groups of place fields differ? We first examined the averaged response profiles 269 for the fields in each group, and found that the BTSP-like fields exhibited strongly forward shifted 270 activity on the formation lap, and relatively reduced amplitude firing on later laps, while the Other ${ }^{271}$ fields were well aligned across laps and fired with more consistent amplitude (Figure 5B). We ${ }_{272}$ quantified the amplitude change by computing a formation lap gain for each place field, and found 273 that the BTSP-like fields consistently exhibited higher gain (Figure 5C). The two clusters were ${ }^{274}$ distinguished by several other features: by construction, the BTSP-like fields exhibited greater 275 shifts in their formation lap activity (Figure S8A), but they also generally exhibited greater place 276 field width on later trials and reduced lap-to-lap stability compared to Other fields (Figures S8B,C). 277 To quantify the isolation of the clusters, we constructed a linear classifier to identify place fields as 278 either BTSP-like or other based on these post hoc characteristics (formation lap gain and shift, 279 field width, and stability) and achieved high accuracy across all mice (85\% on average, Figures 280 S8D,E). Further, the clustering identified a highly consistent fraction of place fields as BTSP-like in 281 each mouse (Figure S7C).

Since we could reliably identify a subset of BTSP-like place fields in the dataset, we asked how ${ }^{283}$ the fraction of place fields forming with these dynamics changes as a function of experience (Figure 284 5D). On most days and trial blocks, the fraction of BTSP-like fields was below $40 \%$ of all fields, but 285 on the first exposure to the novel context, this increased to nearly 60\% on average (Figure 5D). The ${ }_{286}$ enrichment of BTSP-like place field formation was also experience dependent, decaying over the ${ }^{287}$ course of the three day experiment sequence. The fraction of fields classified as BTSP-like in each 288 condition also correlated with the strength of the correlation between velocity and field width 289 (Figure 5E) and the distance of population tuning drift during the initial trials in each context 290 block (Figure 5F). Overall, our field classification analysis is in good agreement with the 291 population-level measures of BTSP prevalence, and lends further credence to an increased rate of 292 
BTSP-mediated acquisition of feature tuning during the initial encounters with novel experiences. 293

Our data delineated a transient period of enriched, BTSP-like place field formation during novel 294 learning, but it is possible that these dynamics do not affect all components of new experiences 295 homogeneously. Numerous prior works have detailed how hippocampal representations are biased 296 by the presence of salient cues (Bourboulou et al. 2019) and reinforcement (Hollup et al. 2001, 297 Zaremba et al. 2017, Dupret et al. 2010); these effects may be driven in part by the engagement of 298 different plasticity mechanisms. Examining the spatial distribution of place field formation events, 299 we found that BTSP-like and Other fields tended to concentrate in opposing regions of the virtual 300 environments: BTSP-like fields accumulated in the regions between reward zones, while Other 301 fields were particularly enriched near the reward zones (Figure 6A). Notably both of these 302 distributions were correlated with the spatial distribution of velocities (Figure 6B): animals tended 303 to run quickly between reward zones, and reliably decelerated as they approached each reward. It is 304 important to account for this correlation, as our classification of BTSP-like fields is determined 305 principally from the spatial shift in formation lap firing (Figures $455 \mathrm{~S} 8 \mathrm{E}$ ), which will be more 306 difficult to detect when the animal is running at slower velocities.

We asked how much of the variance in each field formation distribution was explainable by the 308 velocity profile of the animal, by training a linear model to predict field density from the spatially 309 filtered velocity (Figure 6C, see Methods). Our model learned a spatial velocity filter for each 310 animal and each field type. For Other cells, the velocity at the current position consistently 311 predicted a strong negative effect on field density, while for BTSP-like cells, field density was 312 positively predicted by velocity across a range of spatial lags. Overall, the velocity models 313 explained a majority of variance in the distribution of field formation events for both field types 314 (Figure 6D, see also Figure S9). In Figure 6E, we plotted the field distributions for each mouse and 315 field type. Subtracting the model-predicted field distributions from these curves, we found that the 316 residual field counts were largely spatially homogenized and centered at zero, effectively removing 317 the effect of the reward zones (Figure 6F). Given the current data and our field classification 318 methods, we were unable to determine conclusively whether BTSP differentially contributed to the 319 spatial distribution of place fields.

The prior analysis focused on the spatial distribution of place field formation events pooled over ${ }_{321}$ 
A
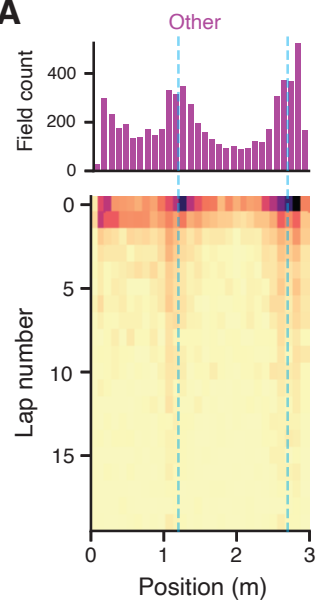

D

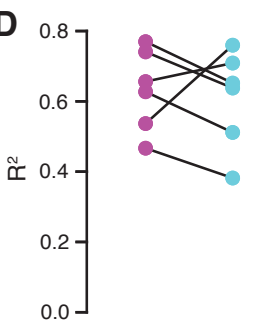

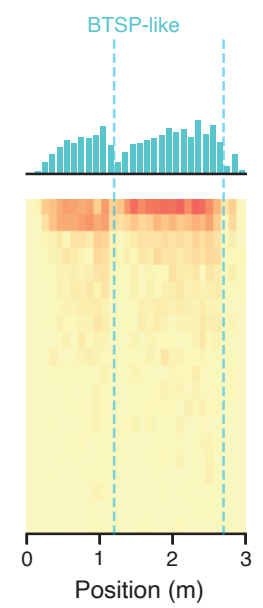

E

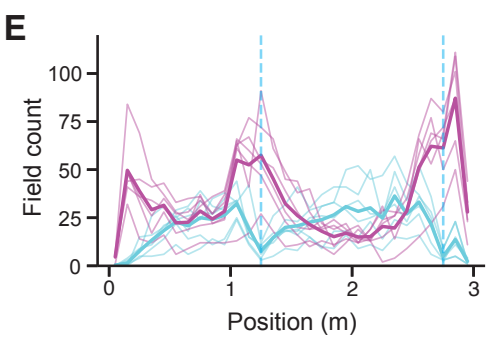

B

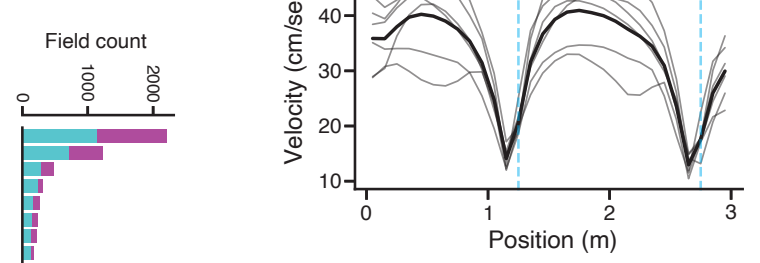

C

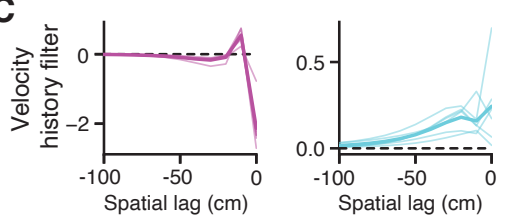

$\mathbf{F}$

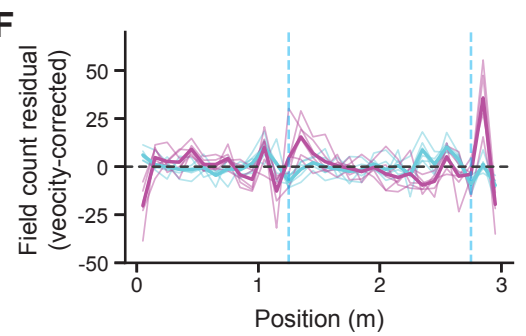

Figure 6. The spatial distribution of place field formation events. A: Histogram of place field formation events over positions and lap number (from the start of the context block), with marginal distributions. Data are pooled from all conditions, shown separately for fields classified as BTSP-like and Other in the preceeding analysis. Dashed blue lines indicate reward zones. B: Average velocity profile across the environment, for each mouse. The mice slow down considerably on approach to the reward zones. C: Spatial kernels fit to predict place field density from the average velocity profile from each mouse. These describe the weighted average of velocity over past and present positions that best predicts the place field density at the present position. Velocity has a large negative effect on Other field density on a short spatial scale. Conversely, BTSP-like field density is positively affected by velocity, on a longer spatial scale. D: Prediction quality of the velocity-to-field density model learned in C. Filtered velocity explains a large fraction of field density variance for both field types. E: As in A, the distribution of place field formation events for Other and BTSP-like fields, shown separately for each mouse. F: As in E, but showing the residual field counts obtained after subtracting off the predictions of the model in $\mathbf{C}, \mathbf{D}$. The residuals are largely spatially homogeneous and centered at zero, reflecting that the majority of variance is explained by velocity.

all trials. On any given trial though, we hypothesized that the locations of new place fields might 322 be constrained by the representation assembled over prior laps. This could arise from the recruitment of lateral inhibition at locations with existing place fields (Rolotti et al. 2021, Milstein 324 et al. 2020, Robinson et al. 2020), promoting competitive interactions that repel accumulating fields 325 away from one another. The result would be that the spatial distribution of new fields on a given 326 lap is not purely random but is also conditional on the location of recently acquired fields. To test ${ }_{327}$ this idea, we examined the formation lap for every place field and measured the average spatial 328 distance between that field and all fields of the same class that formed on the next lap (Figure ${ }_{329}$ 7A,B). Since we were interested in the next-lap field distance that was not explained by the overall 

7B). In Figure 7C, we plotted the distribution of normalized distances for BTSP and Other fields. 333 For both groups of fields, place fields tended to form farther away from fields that formed on the 334 previous lap than would be expected from random sampling of the environment. This history 335 dependence indicates the presence of competitive interactions that act to disperse new place fields, 336 which could help to drive pattern separation of nearby locations.

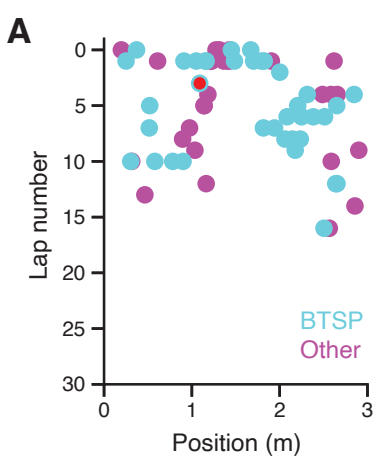

B
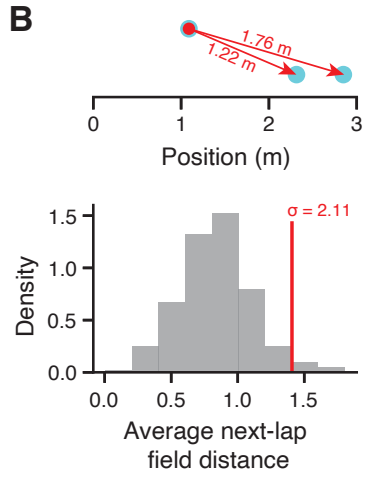

C

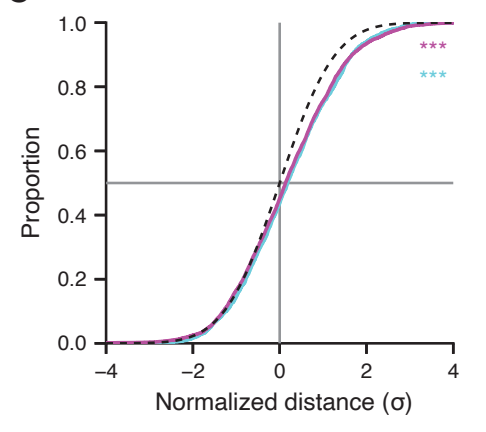

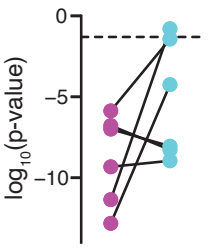

Figure 7. Place field accumulation follows a nonstationary distribution over positions. A: Scatter plot of place field formation event over laps and positions, for an example session (Day 1, first novel context exposure). Events are colored according to their BTSP classification. B: Illustration of next-lap distance calculation. Top: for every place field, the distance was calculated between its location and the location of all place fields that appeared on the next lap (of the same BTSP classification). Bottom: the average next-lap field distance was compared to a null distribution built by randomly permuting the formation laps of all place fields in the trial block. A normalized distance $(\sigma)$ was derived by standardizing the true distance relative to the null distribution. C: Cumulative distribution of normalized distances across the dataset for each place field formation type. The standard normal distribution (chance) is plotted in black. Both BTSP and Other fields show greater next-lap field distances than expected by chance (inset: one-sample Kolmogorov-Smirnov (KS) test against the standard normal distribution). Right: significance of KS test computed for each mouse separately. Place fields accumulated at farther distances compared to prior lap fields than expected by random sampling in nearly all mice and conditions. ${ }^{*} p<0.05,{ }^{* *} p<0.01,{ }^{* * *} p<0.001$

\section{Discussion}

Hippocampal circuits are remarkably plastic, with the ability to construct precise representations of novel experiences with very few exposures (Wilson \& McNaughton 1993). This feature is likely critical to the role of the hippocampus in memory storage: it can serve as a fast learning module that rapidly encodes new information, where it is retained and refined for a short period prior to its 342 transfer to and long-term storage in the cortex. (McClelland et al. 1995, Roxin \& Fusi 2013). The 343 recent discovery of BTSP is a striking demonstration of the speed of synaptic learning in the 344 hippocampus (Bittner et al. 2015, 2017), and this mechanism could be a crucial component 
enabling the circuit's mnemonic functions. In our work here, we have quantified the impact of this 346 novel plasticity rule at the scale of large neuronal populations, and tested a differential role in 347 encoding familiar and novel experiences. Considering multiple lines of analysis, our results are 348 consistent with the hypothesis that a sizable fraction of de novo place fields form via BTSP, and 349 that the probability of BTSP events is regulated by the novelty of ongoing experience.

Other reports have questioned the ubiquity of plateau-dependent plasticity events during place 351 field formation (Cohen et al. 2017, Dong et al. 2021). New place fields can appear in a familiar or 352 novel environment in the absence of plateau-associated complex spiking (Cohen et al. 2017), and 353 CA1 neurons can exhibit latent, presynaptic spatial tuning (Lee et al.|2012), which could possibly 354 be amplified to suprathreshold place fields through other synaptic learning processes McKenzie 355 et al. 2021). In our experiments, we detected signatures of BTSP-like place field formation in all 356 conditions, both at the level of neural ensembles (Figures 2 3 ) and single neurons (Figures 4 5). 357 While prior work on BTSP used whole cell patch recordings to unambiguously identify plateau ${ }_{358}$ potentials, our population-scale approach necessitates that we identify these events through 359 secondary characteristics, namely burst firing and shifted spatial tuning on the first lap of place 360 field appearance. These features are derived respectively from the prolonged somatic depolarization 361 induced by the putative plateau potential (Epsztein et al. 2011, Bittner et al. 2015) and the 362 asymmetric plasticity kernel of BTSP (Bittner et al.|2017), and can be measured through functional 363 calcium imaging at the soma. While these features are indirect, our method allowed us to study 364 place field dynamics at scale and compare the relative frequency of field formation events as animals 365 became increasingly familiar with new environments over days, questions that are impossible to 366 tackle with intracellular recordings limited to single neuron preparations (Bittner et al. 2015, 2017). 367

All features of BTSP in the dataset exhibited strong experience-dependent effects, with an 368 enrichment of BTSP-related dynamics specifically during the first exposures to the novel 369 environment that decayed over subsequent days. Of course, it is obvious that over multiple days of 370 experience in the same environment, we should expect progressively fewer place fields to appear via 371 ongoing plasticity, with much of the representation simply recalled due to prior learning (although 372 representations continue to drift in familiar environments, see Ziv et al. (2013)). However, our data 373 indicate that burst-dependent plasticity is particularly important during early learning in a novel 374 
experience, where our classification estimates that the majority of new place fields appear with 375 BTSP-like characteristics (Figure 5). Plateau potentials are generated through the convergence of 376 coincident presynaptic inputs that triggers active dendritic conductances in the apical tuft 377 (Takahashi \& Magee 2009), but the dendritic arbor of CA1 pyramidal neurons is also extensively 378 regulated by local feedback inhibition (Royer et al. 2012, Lovett-Barron et al. 2012). These circuits 379 actively limit dendritic electrogenesis and tightly restrict the number of place fields that can be 380 simultaneously induced via BTSP (Rolotti et al. 2021). This result implies that other factors must 381 be present that transiently free the circuit of these constraints, in order to rapidly build new 382 representations during novel experience.

There is already evidence for a temporary reduction in dendritic inhibition during novel experience in the hippocampus (Sheffield et al. 2017, Geiller et al. 2020). We hypothesize that these 385 inhibitory dynamics are at least partially regulated by factors originating outside of the 386 hippocampus, such as neuromodulatory inputs from the basal forebrain or brainstem 387 (Palacios-Filardo \& Mellor 2019) that could be involved in broader, brain-wide signaling of novelty 388 detection. In particular, the locus coeruleus is highly sensitive to novelty and provides dense 389 dopaminergic and noradrenergic input to CA1 (Takeuchi et al. 2016), and these projections have 390 been optogenetically manipulated to increase place field density during a reward learning behavior 391 in mice (Kaufman et al. 2020). Cholinergic neurons are also known to respond to reinforcement and 392 are sensitive to stimulus uncertainty (Hangya et al. 2015), and cholinergic projections from the 393 medial septum ramify extensively in CA1, where they are thought to modulate network function 394 and plasticity in response to arousal (Teles-Grilo Ruivo \& Mellor 2013). Characterization of these 395 inputs in vivo remains a relatively nascent endeavor, and given the diversity of receptor expression 396 in the hippocampal circuit (Teles-Grilo Ruivo \& Mellor 2013), it is likely that they affect both the 397 dendritic excitability of pyramidal neurons and also the recruitment of distinct inhibitory 398 microcircuits. In future work, it will be necessary to assess how neuromodulatory projections affect 399 the generation of plateau potentials and facilitate learning during novel exploration. Overall, we 400 hypothesize that the interplay of external novelty signals and regulation of local inhibition may 401 provide a temporary window of hyper-excitability during new experience, which may permit rapid 402 assembly of representations through BTSP. Abstractly, the temporary shift to frequent 
burst-mediated place field formation may contribute to an adaptive learning rate in the

hippocampus, where the circuit responds to a large change in the input statistics by scaling up the ${ }_{405}$ speed of synaptic updates in order to encode new information.

Neuromodulators are often thought to exercise global effects on network state, but other CA1 neurons, either through changes to intrahippocampal afferents (Zhao et al. 2020) or cortical ${ }_{410}$ inputs (Boccara et al. 2019, Butler et al. 2019). This upstream reorganization could also occur in ${ }_{411}$ response to salience or reinforcement, and serve as an instructive signal for guiding hippocampal $\quad 412$ plasticity (Milstein et al. 2020). Targeting plasticity to a fraction of the network is one strategy to 413 negotiate the stability-plasticity dilemma and prevent overwriting older memories too quickly. ${ }_{414}$

We found that place fields forming via BTSP were generally less precise than the remaining 415 fields (Figure S8). While many neurons may first reach threshold via BTSP, it seems likely that ${ }_{416}$ this initial tuning is refined through secondary mechanisms, such as conventional Hebbian rules and 417 local competitive interactions with other pyramidal neurons mediated through lateral inhibition ${ }_{418}$ (Mehta et al. 2000, Cohen et al. 2017, McKenzie et al. 2021, Robinson et al. 2020). Considering ${ }_{419}$ that many of the non-BTSP fields are pre-existing place fields learned during previous experience, ${ }_{420}$ this could explain why BTSP fields were consistently wider, having not yet undergone further ${ }_{421}$ refinement. This is also compatible with the theory that the hippocampus is actively learning a ${ }_{422}$ compressed, decorrelated representation of the environment (Gluck \& Myers 1993, McClelland et al. 423 1995, Schapiro et al. 2017, Benna \& Fusi 2016), as narrower place fields will decrease the correlations between nearby, similar locations. In line with this argument, we also found that the accumulation of place fields was history-dependent (Figure 7). New fields tended to appear at 425 locations farther away from those forming on the previous lap, suggesting that local CA1 networks representation and minimize the neural correlation between nearby locations.

Earlier studies reported that many CA1 place fields undergo a transient period of backward, asymmetric expansion during initial traversals of an environment (Mehta et al. 1997), an effect that ${ }^{431}$ encoded the direction of travel and could be modeled by plasticity at feedforward CA3-to-CA1 
synapses (Mehta et al. 2000). In our experiments, animals were constrained to run in a single 433 direction through the track, and so it is possible that some component of field width and drift is $\quad{ }_{434}$ due to these effects as well, especially residual drift on the trials immediately after place field ${ }_{435}$ formation (Figure S4). However, this does not explain the exaggerated first lap shifts and firing $\quad 436$ rate gains observed in BTSP-like cells nor the enrichment of these patterns during novel ${ }_{437}$ exploration, as the asymmetric expansion reported by Mehta et al. (1997) occurred in both familiar ${ }^{438}$ and novel environments. Due to the sensitivity of fluorescence calcium indicators, it is also likely ${ }^{439}$ that we cannot detect very low firing rate activity and so our ability to resolve any asymmetric $\quad 440$ expansion is limited. More recently, Dong et al. (2021) reported pervasive and continual backward ${ }_{441}$ drift of CA1 place fields in virtual reality. However, the shifts in field locations in our data were ${ }_{442}$ overwhelmingly limited to the first few laps following place field formation (Figures 3 4 S4), which ${ }_{443}$ agrees with prior work in freely moving animals where individual place fields do not continuously ${ }_{444}$ drift (Frank et al. 2004, Mehta et al. 2000).

While here we focused on overall environmental novelty, the hippocampal place code is also Bourboulou et al. 2019) and the presence of reinforcement (Hollup et al. 2001, Zaremba et al. 2017, 448

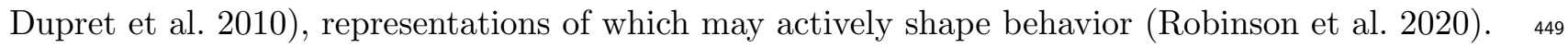
We searched for evidence of differential concentration of BTSP-like field formation along the virtual 450 track, but found that the distribution was mainly uniform over space after regressing out the ${ }_{451}$ component correlated with velocity (Figure 6). In our experiment, the location of reward is highly 452 confounded with the velocity profile of the animal, and so we cannot determine concretely which is ${ }_{453}$ the causal factor for the variability in field distributions. Since the field shift associated with BTSP ${ }_{454}$ is most apparent at higher running speeds and this is a critical component of our field classification 455 method, we certainly underestimate the rate of BTSP events that occur near the reward zones, and 456 so our results do not preclude a connection between burst-dependent plasticity mechanisms and ${ }_{457}^{457}$ reinforcement (Milstein et al. $\mid 2020)$.

Our results are congruent with burst-dependent plasticity as an important contributor to 
consequences this would have, given the differing circuit architecture (Van Strien et al. 2009). In 462 CA1, pyramidal neurons receive a convergence of inputs from intrahippocampal recurrent networks ${ }_{463}$ in CA3, which are believed to store memories through attractor dynamics (Rolls 2007), and from ${ }_{464}$ the superficial entorhinal cortex, which can directly relay sensory information about ongoing 465 experience. Novelty detection may be central to the function of CA1 within the broader ${ }_{466}$ hippocampal circuit (McClelland et al. 1995, Lisman \& Otmakhova 2001). We suggest that the ${ }_{467}$ regulation of burst-dependent plasticity in CA1 may selectively permit the integration of novel ${ }_{468}$ information into the hippocampus, instigating a cascade of plasticity throughout the 469 hippocampal-cortical loop that could optimize internal representations to adapt to large changes in 470 the statistics of ongoing experience.

\section{Acknowledgments}

We thank Zhenrui Liao, Pamela Rivière, and Nick Robinson for helpful discussions, and for

\section{Author Contributions}

J.B.P., S.F., and A.L. conceived the project. J.B.P. designed experiments, collected data, and ${ }^{481}$ performed analysis and modeling. J.C.B. built the virtual reality and behavioral control systems, ${ }^{482}$ with assistance from J.B.P. S.V.R. provided critical input on analysis. J.B.P., S.F., and A.L. wrote ${ }^{483}$ the paper with input from all authors.

\section{Declaration of Interests}




\section{Lead Contact and Materials Availability}

Further information and requests for resources and reagents should be directed to the Lead Contact 489 Attila Losonczy (al2856@columbia.edu). All unique resources generated in this study are available 490 from the Lead Contact with a completed Materials Transfer Agreement.

\section{Experimental Model and Subject Details}

All experiments were conducted in accordance with the NIH guidelines and with the approval of the ${ }_{493}$ Columbia University Institutional Animal Care and Use Committee. Experiments were performed 494 with adult (8-16 weeks) male C57Bl/6 mice (Jackson Laboratory).

\section{Method Details}

\section{Behavior and Imaging}

Viruses Pyramidal cell imaging experiments were performed by injecting a recombinant 498 adeno-associated virus (rAAV) encoding GCaMP6f (rAAV1-Syn-GCaMP6f-WPRE-SV40,

Surgical procedure Methods for viral delivery and surgical implant of imaging window and 501 headposts were largely identical to previous work (Lovett-Barron et al.|2014). Briefly, mice were 502 anesthetized under isofluorane and the virus was injected in dorsal CA1 (-2 mm AP; -1.5 ML, -1.2 503 DV relative to bregma; $500 \mathrm{~nL}$ ) using a Nanoject syringe. Mice recovered in their home cage for 3504 days following viral infusions. We then aspirated the cortex overlying the left dorsal hippocampus 505 and implanted a $3 \mathrm{~mm}$ glass-bottomed stainless steel cannula for imaging access, and cemented a 506 titanium headpost to the skull for head-fixation. For all surgeries, monitoring and analgesia 507 (buprenorphine or meloxicam as needed) was continued for 3 days postoperatively.

Behavioral apparatus and virtual reality system Mice were head-fixed above a low-friction, 509 light weight running wheel (Warren et al. 2021). The axle of the running wheel was coupled to a 510 
rotary encoder, which connected to a circuit that decoded and buffered the quadrature data from 511 the encoder and transmitted these position updates to an Intel NUC i5 mini-PC that was used to 512 control the behavior system. As described previously (Kaufman et al. 2020), the experiments were 513 managed through custom software on the PC that would send and receive instructions from a 514 custom GPIO circuit on the behavior apparatus, which managed the water delivery system, lick 515 port sensor and synchronization with the imaging system, as well as from the position tracking 516 circuit and from the virtual reality system. All system elements were connected using high-speed 517 Ethernet and communicated via UDP message passing.

The running wheel was surrounded by 5 LCD computer monitors (Acer SB230 23" IPS screens) 519 arranged in a half-octagon, covering approximately $220^{\circ}$ of the visual field of the mouse. Each 520 monitor was connected to an individual ODROID-C2 single board computer running the Android ${ }_{521}$ operating system (version 6.0.1) which rendered a fraction of the VR scene on each display. Each 522 ODROID received continuous instructions over Ethernet from the behavior control computer to ${ }_{523}$ update the VR environment as the position of the animal advanced. Virtual reality scenes were 524 designed using the Unity game engine.

Behavior training Starting 7 days after implant surgery, mice were habituated to handling and 526 head-fixation as previously described (Lovett-Barron et al. 2014). After two days of acclimation 527 and free running on the wheel, we began exposing the animal to the $3 \mathrm{~m}$ virtual environment. The 528 environment used during this training period would later become the "Familiar" context for the ${ }_{529}$ main experiment. When the mice reached the end of the virtual track, the screens were 530 momentarily blanked for a 2 sec inter-trial interval, after which they were instantly teleported back 531 to the beginning of the track.

At this point, mice were water deprived to $85-90 \%$ of their starting weight. Over a period of 1-2 533 weeks, we trained mice to run forward through the virtual environment and lick for small volume 534 sucrose solution rewards (5\% sucrose, $\sim 4 \mu \mathrm{m}$ per reward). Rewards were initially dispersed 535 randomly throughout the environment to encourage running, and we slowly reduced the reward 536 count to two fixed reward zones (located at $\sim 1.2$ and $2.7 \mathrm{~m}$ on the track) over the training period. 537 Mice reached training criteria when they could consistently run > 130 laps using the two fixed 538 
reward zones in under an hour. Mice were given additional water as needed daily to maintain 539 weights.

Familiar-Novel context switching paradigm For context-switch experiments, mice ran 541 through alternating blocks of trials in the Familiar (training) context and a Novel context that was 542 previously unseen prior to the start of the imaging experiments. Each recording session was 543 organized into 4 blocks: 40 trials in Familiar, 30 trials in Novel, 30 trials in Familiar, and 30 trials 544 in Novel. We additionally repeated this experiment with the same contexts across two additional 545 days, and so a complete experiment sequence represented 3 days of recording. In all trials and 546 contexts, the distance to the sucrose rewards remained fixed at 1.2 and $2.7 \mathrm{~m}$. For each mouse, we 547 also repeated the entire experiment sequence with a second Novel context (the familiar context 548 remained the same for both sequences).

2-photon microscopy Mice were habituated to the imaging apparatus (e.g. microscope/objective, laser, sounds of resonant scanner and shutters) during the training period. immersion objective (Nikon, 0.8 NA, 3 mm working distance). For excitation, we used a $920 \mathrm{~nm}{ }^{553}$ laser (50-100 mW at objective back aperture, Coherent). Green (GCaMP6f) fluorescence was 554 collected through an emission cube filter set (HQ525/70 m-2p) to a GaAsP photomultiplier tube ${ }_{555}$ detector (Hamamatsu, 7422P-40). A custom dual stage preamp $(1.4 \times 105 \mathrm{~dB}$, Bruker) was used to 556 amplify signals prior to digitization. All experiments were performed at 1.2-2x digital zoom, 557 acquired as $512 \times 512$ pixels images at $10 \mathrm{~Hz}$.

\section{Quantification and Statistical Analysis}

Image preprocessing Imaging data was organized using the SIMA software package Kaifosh 560 et al. 2014). Data was motion corrected in Suite2p (Pachitariu et al. 2017) using the non-rigid 561 registration mode. ROIs were also detected using Suite2p (using "sparse mode"), followed by 562 Suite2p's standard fluorescence extraction and neuropil correction. Identified ROIs were curated 563 post-hoc using Suite2p's graphical interface to exclude non-somatic components. 


\section{Neural data analysis}

Event detection All fluorescence traces were deconvolved to detect putative spike events, using 566 the OASIS implementation of the fast non-negative deconvolution algorithm (Friedrich et al. 2017). 567 As in Ahmed et al. (2020), we discarded any events whose amplitude was below 4 median absolute 568 deviations of the raw trace, and discretized the resulting signal for all subsequent analysis.

Calculating spatial tuning curves Spatial tuning curves were calculating for each neuron on 570 each lap. The virtual track was discretized into 100 evenly spaced bins $(3 \mathrm{~cm})$, which were used to 571 compute a histogram of neural events. After normalizing for animal occupancy, the histogram was 572 convolved with a Gaussian kernel $(\sigma=9 \mathrm{~cm})$ to obtain a smooth activity rate estimate. concatenating the spatial tuning curves of all neurons in the population. Similarly, we obtained the 577 spatial cross-correlation between the two trials across a range of spatial lags by first shifting each 578 neuron's spatial tuning in trial $b$ and then recomputing the population vector correlation with $a$. 579 Here a peak in the spatial cross-correlation at a positive lag indicates that the activity of $a$ is ahead 580 of $b$ in space (since $b$ must be shifted forward to maximize the correlation), and conversely, a peak 581 at a negative lag indicates that activity in $a$ is generally at locations behind $b$. We summarized the 582 directionality and distance of the shift between trial pairs by the center-of-mass (COM) of the ${ }_{583}$ spatial cross-correlation curve.

The pattern of spatial drift across trials can be visualized by plotting the spatial shifts for all 585 pairs of trials as a matrix. The matrix is necessarily anti-symmetric (since the cross-correlation 586 between $a$ and $b$ is the mirror image of that between $b$ and $a$ ). Different neural dynamics predict $\quad 587$ qualitatively different shift matrices: a population of continually drifting place cells would exhibit a 588 diagonally-banded shift matrix (since shift is then a monotonic function of the time between trials), 589 while a more transient population shift (for example, during a period with many place fields 
The shifting tendency of each individual trial relative to the whole trial block can be determined 593 by averaging the rows (or columns) of the symmetrized shift matrix (i.e. by multiplying the matrix 594 lower triangle by -1). Symmetrizing is necessary so that all shift comparisons for a given trial 595 consistently indicate the direction of movement. For example, if the neural population is 596 consistently shifting backward, trials before trial $n$ will be ahead and trials after will be behind. $\quad{ }_{597}$ Averaging the $n$th row of the symmetrized matrix is equivalent to averaging all trial comparisons in 598 the matrix upper triangle that include trial $n$. By our convention, a positive average shift for trial $n 599$ indicates it is part of a generally backward trend, while a negative average shift indicates a forward 600 trend, relative to the surrounding trials in the block. Occasionally activity on some trials correlated 601 poorly with the rest of the block (possibly due to lapses in attention or behavior), and so we 602 excluded any trial pairs where the peak of the spatial cross-correlation was less than 0.1. This ${ }_{603}$ method is used to calculate the average shifts in Figure 2C and S?, where we additionally omitted 604 any trials in an experiment where $>1 / 3$ rd of trial pair comparisons were missing due to this ${ }_{605}$ exclusion criteria.

Simulations of place field accumulation We validated the population cross-correlation on simulated datasets of accumulating place fields, where a sub-fraction of fields exhibited BTSP-like 608 characteristics (first-lap shift and gain), or that exhibited linear drifting over trials. Each simulated ${ }_{609}$ population comprised 300 neurons, where each neuron had a 0.2 probability of acquiring a place ${ }_{610}$ field. Fields accumulated over 30 laps according to a geometric process with mean 6, which is ${ }_{611}$ approximately the observed average first lap for place fields during the first exposure to the novel ${ }_{612}$ context during Day 1 of the experiment. Place field centers were sampled uniformly over the ${ }_{613}$ environment, and activity was modeled on each lap as a Gaussian bump ( $\sigma=5$ bins) at the ${ }_{614}$ sampled location.

For simulations of BTSP, we fixed a probability of BTSP for place fields in each simulation. If a ${ }_{616}$ field was drawn as BTSP, we shifted its first lap activity by a random distance sampled uniformly ${ }_{617}$ between 0 and 0.25 of the environment length, and scaled its first lap activity by a random gain ${ }_{618}$ sampled uniformly between 1 and 3. We then considered the analysis results for different BTSP ${ }_{619}^{619}$ probabilities. 
For simulation of continuously drifting place fields, we fixed a probability of drifting for place ${ }_{621}$ fields in each simulation. If a field was drawn as drifting, we sampled a slope (distance-by-trial) for 622 the drift uniformly from X to X, and shifted each lap's activity according to that drift function. We ${ }_{623}$ then considered the analysis results for different drifting probabilities.

For all neurons, we additionally added out-of-field noise to individual laps with a probability of 625 0.2. This was simulated as additional Gaussian bumps added to that lap's activity, centered at a ${ }_{626}$ random location in the environment. We additionally applied random scaling noise to each lap, ${ }_{627}$ with each lap's gain drawn $\sim \mathcal{N}(1,0.05)$.

Place field detection We detected place fields separately in each context trial block (4 per spatial occupancy. We constructed a null distribution of spatial tuning curves for every neuron, by 632 circularly shifting each lap's activity independently by a random distance, and recomputing the smoothed, lap-averaged tuning curve as described previously. This procedure was repeated 1000 times, and we determined the 95th percentile of null tuning values at every spatial bin (i.e. the exceeded this null threshold were marked as candidate place fields, and the place field width was calculated as the distance between where the true tuning curve first exceeded and then again fell below the threshold curve. To restrict our analysis to neurons with unambiguous firing fields, we ${ }_{639}$ additionally required that place fields have a width $<1 \mathrm{~m}$ (i.e. $\frac{1}{3}$ of the track), and that the neuron ${ }_{640}$ is active within the bounds of the place field on at least 15 laps.

Spatial tuning score We computed a pseudo-probability mass function $p\left(x_{i}\right)$ for each spatial tuning curve, where $x_{1}, x_{2}, \ldots x_{n}$ are the discrete position bins, by normalizing the tuning curve so ${ }_{643}^{64}$ that it sums to 1 over positions. We then compute the un-normalized tuning score $\hat{s}$ as the

$$
\hat{s}=\mathrm{D}_{K L}(p \| u)=\log _{2} n+\sum_{i=1}^{n} p\left(x_{i}\right) \log _{2} p\left(x_{i}\right)
$$


Intuitively, uniform activity over space will give the minimum score $s=0$, while having all 646 activity concentrated in a single position bin will yield the maximum score of $s=\log _{2}(n)$. Since ${ }_{647}$ inhomogenous spatial tuning can also arise simply from very sparse, noisy activity, in practice we ${ }_{648}$ used a normalized tuning score $s$ by standardizing $\hat{s}$ relative to a null distribution, formed by ${ }_{649}$ calculating the tuning score on all null tuning curves (generated as described in the place field 650 $\begin{array}{ll}\text { detection procedure): } & 651\end{array}$

$$
s=\frac{\hat{s}-\mu\left(\hat{s}_{\text {null }}\right)}{\sigma\left(\hat{s}_{\text {null }}\right)}
$$

Detecting the lap of place field formation For our analysis of place field characteristics, it ${ }_{652}$ was necessary to determine the first lap that a neuron fired within its place field(s) in a given trial 653 block. Following Sheffield et al. (2017), we identified for each place field the first window of 5 laps 654 where the neuron was active within the place field boundaries on at least 3 of those laps, and called 655 the first active lap within that window the place field formation lap. A lap was considered active if 656 there was activity within the place field boundary on that trial with amplitude of at least $5 \%$ of the ${ }_{657}$ neuron's peak activity level across all trials. Our results however did not strongly depend on the ${ }_{658}$ particular choice of thresholds.

Formation lap activity displacement We estimated the spatial shift between activity on the ${ }_{660}$ lap of place field formation and activity on the remaining laps within the place field. This was ${ }_{661}$ computed as the difference between the location of peak firing on the first lap, and the location of ${ }_{662}$ peak firing in the average tuning of all subsequent laps (as in the population shift analysis, a ${ }_{663}^{6}$ positive shift indicates the formation lap's activity was at positions ahead of the later lap's activity). ${ }_{664}$

Formation lap gain Evidence for elevated activity or burst-firing during the formation lap was 665 assessed by computing the formation lap gain, defined as the peak activity rate on the formation $\quad{ }_{666}$ lap within the place field divided by the peak activity rate in the averaging tuning of all subsequent ${ }_{667}$ laps that contained activity within the place field (i.e. excluding any silent laps, so that the gain ${ }_{668}$ does not simply reflect unreliable place fields). A gain $>1$ indicates elevated firing during the place ${ }_{669}$ field formation lap, relative to later traversals through the place field. 
Peri-field formation velocity For each place field, we computed the average velocity of the $\quad{ }_{671}$ animal within the place field boundaries during the place field formation lap.

Velocity-field width correlation For each trial block on each day, we fit a linear model to ${ }_{673}$ predict the width of place fields from the peri-field formation velocity. We compared the slope of ${ }_{674}$ the linear fits between Familiar and Novel contexts by calculating a $\Delta$ slope (Novel - Familiar) for 675 each context switch. We calculated the probability that this $\Delta$ would be observed under random ${ }_{676}$ permutations of the context labels for place fields, recomputing $\Delta$ slope for 10000 shuffles in each 677 condition. A p-val was obtained from the cumulative density of the Gaussian fit to the resulting 678 null distribution. We reported the significance relative to the null distribution as the negative 679 $\log _{10}$ (p-val). In Fig. 3F, G, we pooled place fields across all experiments from a given day and trial 680 block. In Fig. S5, these analyses were repeated for each mouse separately, pooling data from the ${ }_{681}$ two repetitions of the experiment sequence for each mouse to reach adequate place field counts. ${ }_{682}$

Non-negative matrix factorization We aimed to identify subsets of place fields in the dataset ${ }_{683}$ that exhibited different kinds of trial-to-trial dynamics (e.g. BTSP-like shift in the formation lap ${ }_{684}$ activity, drifting responses, or amplitude modulation over trials). To this end, we first aligned all ${ }_{685}$ place field tuning profiles by extracting a $150 \mathrm{~cm}$ (50 bins) window of activity from each lap around 686 the place field center, and concatenating these windows for the first 10 laps from the formation lap ${ }_{687}$ of the place field (e.g. Fig. 4A). This yielded a 500 element vector $\mathbf{x}$ for every place field, which we ${ }_{688}$ stacked into a matrix $\mathbf{X}$ so that each row was the spatiotemporal profile (trial $\times$ position) of a ${ }_{689}$ single place field.

Our goal then was to cluster the rows of $\mathbf{X}$ in order to identify groups of place fields with similar 691 trial-to-trial dynamics, but clustering samples directly in high-dimensional spaces is generally a poor ${ }_{692}$ strategy. Instead, we first reduced the dimensionality of $\mathbf{X}$ using non-negative matrix factorization: ${ }_{693}$

$$
\mathbf{W}, \mathbf{H}=\underset{\mathbf{W}, \mathbf{H}}{\operatorname{argmin}}\|\mathbf{X}-\mathbf{W H}\|_{\text {Fro }}^{2}
$$

Where $\mathbf{W}$ and $\mathbf{H}$ are rank $n$ matrices, for some $n \ll 500$. $\mathbf{H}$ is an $n \times 500$ matrix, where each of ${ }_{694}$ the $n$ rows is a spatiotemporal pattern. NMF models each row (place field) in $\mathbf{X}$ as a weighted sum ${ }_{695}$ 
of the these $n$ patterns, given by $\mathbf{W}$. As with any dimensionality reduction method, since $n$ is much 696 less than the number of place fields in the dataset, the model is forced to identify spatiotemporal ${ }_{697}$ patterns in $\mathbf{H}$ that are shared between many place fields. The strict non-negativity of $\mathbf{H}$ and $\mathbf{W}$ in ${ }_{698}$ NMF however can often give particularly interpretable decompositions, due to its parts-based 699 reconstruction of $\mathbf{X}$ (i.e., since all elements in the weighted sum for each place field are 700 non-negative, the different patterns in $\mathbf{H}$ cannot "cancel out"). Each row of $\mathbf{X}$ was 701 mean-normalized prior to decomposition, so the optimization was not dominated by inhomogenous 702 activity scales across neurons or experiments.

We inspected NMF decompositions of the data for a range of $n$, and found that $n=11$ corresponded to a prominent "elbow" between two linear regimes in the loss function (i.e. the rate 705 of improvement slows when adding any additional components $>11$, Fig. 4 C). Additionally, $n=11706$ consistently gave the most interpretable spatiotemporal patterns in $\mathbf{H}$ (Fig. 4D, S6). In particular, 707 this model produced components that separately represented the place field on each of the 10 laps, 708 plus an additional forward-shifted component on the first lap, reminiscent of plateau-driven place 709 field formation. The weights of the two first-lap components among place fields were strongly 710 anti-correlated (Fig. S6).

Clustering place fields in NMF space We used K-means clustering with $K=2$ to partition 712 place fields into "BTSP-like" and "other" groups, using the 11 NMF patterns as the feature space 713 for clustering. We found that 2 clusters were sufficient to reliably segregate place fields with 714 BTSP-like characteristics. In particular, we found that increasing the number of clusters had 715 negligible effects on the BTSP-like cluster; additional clusters formed largely through additional $\quad 716$ subdivisions of the "other" group (Fig S7).

BTSP fraction We computed the fraction of place fields in each experimental condition (day, ${ }_{718}$ trial block) that were assigned to the BTSP-like group. In Fig. 4D, we computed this per 719 experimental session. In Fig. $4 \mathrm{E}, \mathrm{F}$, this was computed by mouse (pooling each mouse's data from 720 the two repetitions of the experiment, as was done for Fig $\mathrm{S} 5$. 
Decoding We validated the NMF-clustering analysis by attempting to decode the BTSP/Other 722 labels of place fields based on secondary characteristics of their activity profiles. We computed $4{ }_{723}$ features for every place field in the dataset: the shift in its tuning from the first lap to remaining 724 laps, the width of the place field, the stability of its place field (correlation between even and odd 725 laps), and its first lap activity gain. We then trained a support vector machine with a linear kernel 726 to classify place fields as BTSP or Other in this 4-dimensional feature space (Fig. S8). We reported ${ }_{727}$ an averaged cross-validated decoding accuracy for each mouse, by randomly partitioning the data 728 into 10 50/50\% training/test splits, stratified by BTSP label. Samples were weighted during 729 training to be inversely proportional to label frequency to account for the greater number of Other 730 cells. We additionally compared the cross-validated results to a null distribution constructed by 731 rerunning the cross-validated decoding analysis on copies of the dataset where the BTSP 732 classifications of place fields were randomly permuted. This procedure was repeated 1000 times, 733 and significance thresholds were computed from a $95 \%$ interval on the resulting distribution of null 734 accuracies.

Modeling the effects of velocity on place field density We fit a linear model to predict place field density at each position as a function of the animal's spatial velocity profile. The predictors in the model were exponentially filtered versions of the spatial velocity profile $(\tau \in\{0.01,0.025,0.05,0.1,0.25\}$ spatial bins $)$, to account for any short-term history effects, e.g. $\quad 739$ those induced by the calcium autocorrelation. The velocity filters plotted in Figure 6C are obtained 740 by a weighted sum of the exponential kernels, with the weights given by the regression coefficients. 741

Next-lap field distance To test for history dependence in the locations of accumulated place 742 fields, we computed for every place field $n$ its average distance to place fields that formed on lap 743 $t+1$, where $t$ is the formation lap for field $n$. These distances were computed only between fields of 744 the same BTSP classification. We then standardized this distance according to a null distribution, 745 obtained by randomly permuting the lap of place field formation between all fields of a given BTSP 746 classification within each trial block. In this way, we disrupt the correlations between adjacent laps 747 while maintaining the marginal distributions of place fields over positions and laps. This procedure 748 was repeated 200 times for each place field. The resulting $\sigma$ measures the next-lap field distance 749 
bioRxiv preprint doi: https://doi.org/10.1101/2021.07.02.450956; this version posted July 3, 2021. The copyright holder for this preprint (which was not certified by peer review) is the author/funder, who has granted bioRxiv a license to display the preprint in perpetuity. It is made available under aCC-BY-NC-ND 4.0 International license.

relative to the distance expected simply from random sampling according to the marginal 


\section{References}

Ahmed, M. S., Priestley, J. B., Castro, A., Stefanini, F., Canales, A. S. S., Balough, E. M., Lavoie, 753 E., Mazzucato, L., Fusi, S. \& Losonczy, A. (2020), 'Hippocampal network reorganization 754 underlies the formation of a temporal association memory', Neuron 107(2), 283-291.

Benna, M. K. \& Fusi, S. (2016), 'Computational principles of synaptic memory consolidation',

Bittner, K. C., Grienberger, C., Vaidya, S. P., Milstein, A. D., Macklin, J. J., Suh, J., Tonegawa, S. 758 \& Magee, J. C. (2015), 'Conjunctive input processing drives feature selectivity in hippocampal 759 ca1 neurons', Nature Neuroscience 18, 1133-1142.

Bittner, K. C., Milstein, A. D., Grienberger, C., Romani, S. \& Magee, J. C. (2017), 'Behavioral 761 time scale synaptic plasticity underlies CA1 place fields', Science $\mathbf{3 7 5}$, 1033-1036.

Boccara, C. N., Nardin, M., Stella, F., O’Neill, J. \& Csicsvari, J. (2019), 'The entorhinal cognitive 763 map is attracted to goals', Science 363(6434), 1443-1447.

Bourboulou, R., Marti, G., Michon, F.-X., El Feghaly, E., Nouguier, M., Robbe, D., Koenig, J. \& 765 Epsztein, J. (2019), 'Dynamic control of hippocampal spatial coding resolution by local visual 766 cues', Elife 8, e44487.

Butler, W. N., Hardcastle, K. \& Giocomo, L. M. (2019), 'Remembered reward locations restructure 768 entorhinal spatial maps', Science 363(6434), 1447-1452.

Carpenter, G. A. \& Grossberg, S. (1991), Pattern recognition by self-organizing neural networks, 770 MIT Press.

Cohen, J. D., Bolstad, M. \& Lee, A. K. (2017), 'Experience-dependent shaping of hippocampal CA1 772 intracellular activity in novel and familiar environments', eLife.

Diamantaki, M., Coletta, S., Nasr, K., Zeraati, R., Laturnus, S., Berens, P., Preston-Ferrer, P. \& 774 Burgalossi, A. (2018), 'Manipulating hippocampal place cell activity by single-cell stimulation in 775 freely moving mice', Cell reports 23(1), 32-38. 
Dong, C., Madar, A. D. \& Sheffield, M. E. (2021), 'Distinct place cell dynamics in ca1 and ca3 777 encode experience in new environments', Nature communications 12(1), 1-13.

Dupret, D., O’neill, J., Pleydell-Bouverie, B. \& Csicsvari, J. (2010), 'The reorganization and reactivation of hippocampal maps predict spatial memory performance', Nature neuroscience 780 $\mathbf{1 3}(8), 995$.

Eichenbaum, H. (2017), 'The role of the hippocampus in navigation is memory', Journal of 782 neurophysiology $\mathbf{1 1 7}(4), 1785-1796$.

Epsztein, J., Brecht, M. \& Lee, A. K. (2011), 'Intracellular determinants of hippocampal ca1 place 784 and silent cell activity in a novel environment', Neuron 70(1), 109-120.

Frank, L. M., Stanley, G. B. \& Brown, E. N. (2004), 'Hippocampal plasticity across multiple days 786 of exposure to novel environments', Journal of Neuroscience 24(35), 7681-7689.

Friedrich, J., Zhou, P. \& Paninski, L. (2017), 'Fast online deconvolution of calcium imaging data', 788 PLoS computational biology 13(3), e1005423.

Geiller, T., Vancura, B., Terada, S., Troullinou, E., Chavlis, S., Tsagkatakis, G., Tsakalides, P., 790 Ócsai, K., Poirazi, P., Rózsa, B. J. et al. (2020), 'Large-scale 3d two-photon imaging of ${ }^{791}$ molecularly identified ca1 interneuron dynamics in behaving mice', Neuron 108(5), 968-983. 792

Gerstner, W., Lehmann, M., Liakoni, V., Corneil, D. \& Brea, J. (2018), 'Eligibility traces and ${ }^{793}$ plasticity on behavioral time scales: experimental support of neohebbian three-factor learning 794 rules', Frontiers in neural circuits 12, 53.

Gluck, M. A. \& Myers, C. E. (1993), 'Hippocampal mediation of stimulus representation: A 796 computational theory', Hippocampus 3(4), 491-516.

Hangya, B., Ranade, S. P., Lorenc, M. \& Kepecs, A. (2015), 'Central cholinergic neurons are rapidly recruited by reinforcement feedback', Cell $\mathbf{1 6 2}(5), 1155-1168$.

Hollup, S. A., Molden, S., Donnett, J. G., Moser, M.-B. \& Moser, E. I. (2001), 'Accumulation of 800 hippocampal place fields at the goal location in an annular watermaze task', Journal of 801 Neuroscience 21(5), 1635-1644. 
Kaifosh, P., Zaremba, J. D., Danielson, N. B. \& Losonczy, A. (2014), 'Sima: Python software for ${ }^{803}$ analysis of dynamic fluorescence imaging data', Frontiers in neuroinformatics $\mathbf{8}, 80$.

Kaufman, A. M., Geiller, T. \& Losonczy, A. (2020), 'A role for the locus coeruleus in hippocampal 805 ca1 place cell reorganization during spatial reward learning', Neuron 105(6), 1018-1026.

Lee, D., Lin, B.-J. \& Lee, A. K. (2012), 'Hippocampal place fields emerge upon single-cell manipulation of excitability during behavior', Science 337(6096), 849-853.

Lisman, J. E. \& Otmakhova, N. A. (2001), 'Storage, recall, and novelty detection of sequences by 809 the hippocampus: elaborating on the socratic model to account for normal and aberrant effects 810 of dopamine', Hippocampus 11(5), 551-568.

Lovett-Barron, M., Kaifosh, P., Kheirbek, M. A., Danielson, N., Zaremba, J. D., Reardon, T. R., 812 Turi, G. F., Hen, R., Zemelman, B. V. \& Losonczy, A. (2014), 'Dendritic inhibition in the ${ }^{813}$ hippocampus supports fear learning', Science 343(6173), 857-863. 814

Lovett-Barron, M., Turi, G. F., Kaifosh, P., Lee, P. H., Bolze, F., Sun, X.-H., Nicoud, J.-F., Zemelman, B. V., Sternson, S. M. \& Losonczy, A. (2012), 'Regulation of neuronal input 816 transformations by tunable dendritic inhibition', Nature neuroscience 15(3), 423-430. 817

Magee, J. C. \& Grienberger, C. (2020), 'Synaptic plasticity forms and functions', Annual review of 818 neuroscience 43, 95-117.

Mankin, E. A., Diehl, G. W., Sparks, F. T., Leutgeb, S. \& Leutgeb, J. K. (2015), 'Hippocampal ca2 820 activity patterns change over time to a larger extent than between spatial contexts', Neuron ${ }_{821}$ 85(1), 190-201.

Manns, J. R. \& Eichenbaum, H. (2009), 'A cognitive map for object memory in the hippocampus', 823 Learning \& memory 16(10), 616-624.

McClelland, J. L., McNaughton, B. L. \& O'Reilly, R. C. (1995), 'Why there are complementary 825 learning systems in the hippocampus and neocortex: insights from the successes and failures of 826 connectionist models of learning and memory.', Psychological review 102(3), 419. 
McKenzie, S., Huszár, R., English, D. F., Kim, K., Christensen, F., Yoon, E. \& Buzsáki, G. (2021), ${ }^{828}$ 'Preexisting hippocampal network dynamics constrain optogenetically induced place fields', 829 Neuron 109(6), 1040-1054.

Mehta, M. R., Barnes, C. A. \& McNaughton, B. L. (1997), 'Experience-dependent, asymmetric 831 expansion of hippocampal place fields', Proceedings of the National Academy of Sciences U.S.A 832 94, 8918-8921.

Mehta, M. R., Quirk, M. C. \& Wilson, M. A. (2000), 'Experience-dependent asymmetric shape of 834 hippocampal receptive fields', Neuron 25, 707-715.

Milligan, G. W. \& Cooper, M. C. (1985), 'An examination of procedures for determining the 836 number of clusters in a data set', Psychometrika 50(2), 159-179. independent of correlated activity', bioRxiv .

URL: https://www.biorxiv.org/content/early/2020/02/05/2020.02.04.934182

Moser, E. I., Kropff, E. \& Moser, M.-B. (2008), 'Place cells, grid cells, and the brain's spatial 842 representation system', Annu. Rev. Neurosci. 31, 69-89.

Muller, R. U. \& Kubie, J. L. (1987), 'The effects of changes in the environment on the spatial firing 844 of hippocampal complex-spike cells', Journal of Neuroscience 7(7), 1951-1968.

Pachitariu, M., Stringer, C., Dipoppa, M., Schröder, S., Rossi, L. F., Dalgleish, H., Carandini, M. \& 846 Harris, K. D. (2017), 'Suite2p: beyond 10,000 neurons with standard two-photon microscopy', ${ }_{847}$ Biorxiv .

Palacios-Filardo, J. \& Mellor, J. R. (2019), 'Neuromodulation of hippocampal long-term synaptic ${ }^{849}$ plasticity', Current opinion in neurobiology 54, 37-43.

Robinson, N. T., Descamps, L. A., Russell, L. E., Buchholz, M. O., Bicknell, B. A., Antonov, G. K., 851 Lau, J. Y., Nutbrown, R., Schmidt-Hieber, C. \& Häusser, M. (2020), 'Targeted activation of 852 hippocampal place cells drives memory-guided spatial behavior', Cell 183(6), 1586-1599. 
Rolls, E. T. (2007), 'An attractor network in the hippocampus: theory and neurophysiology', 854 Learning \& memory 14(11), 714-731.

Rolotti, S., Ahmed, M., Szoboszlay, M., Blockus, H., Gonzalez, K., Sparks, F., Canales, A. S. S. \& 856 Losonczy, A. (2021), 'Inhibitory feedback control of behavioral time scale synaptic plasticity ${ }_{857}$ induction in ca1'. COSYNE. $\quad 858$ URL: http://www.cosyne.org/cosyne21/Cosyne2021_program_book.pdf 859

Roxin, A. \& Fusi, S. (2013), 'Efficient partitioning of memory systems and its importance for 860 memory consolidation', PLoS Comput Biol 9(7), e1003146. 861

Royer, S., Zemelman, B. V., Losonczy, A., Kim, J., Chance, F., Magee, J. C. \& Buzsáki, G. (2012), ${ }^{862}$ 'Control of timing, rate and bursts of hippocampal place cells by dendritic and somatic ${ }^{863}$ inhibition', Nature neuroscience 15(5), 769-775. 864

Schapiro, A. C., Turk-Browne, N. B., Botvinick, M. M. \& Norman, K. A. (2017), 'Complementary 865 learning systems within the hippocampus: a neural network modelling approach to reconciling 866 episodic memory with statistical learning', Philosophical Transactions of the Royal Society B: $\quad{ }^{867}$ Biological Sciences 372(1711), 20160049.

Sheffield, M. E., Adoff, M. D. \& Dombeck, D. A. (2017), 'Increased prevalence of calcium transients 869 across the dendritic arbor during place field formation', Neuron 96(2), 490-504.

Takahashi, H. \& Magee, J. C. (2009), 'Pathway interactions and synaptic plasticity in the dendritic 871 tuft regions of ca1 pyramidal neurons', Neuron 62(1), 102-111.

Takeuchi, T., Duszkiewicz, A. J., Sonneborn, A., Spooner, P. A., Yamasaki, M., Watanabe, M., 873 Smith, C. C., Fernández, G., Deisseroth, K., Greene, R. W. et al. (2016), 'Locus coeruleus and 874 dopaminergic consolidation of everyday memory', Nature 537(7620), 357-362. 875

Teles-Grilo Ruivo, L. \& Mellor, J. (2013), 'Cholinergic modulation of hippocampal network 876 function', Frontiers in synaptic neuroscience 5, 2. 877

Van Strien, N., Cappaert, N. \& Witter, M. (2009), 'The anatomy of memory: an interactive 878 overview of the parahippocampal-hippocampal network', Nature reviews neuroscience 879 $\mathbf{1 0}(4), 272-282$. 
Warren, R. A., Zhang, Q., Hoffman, J. R., Li, E. Y., Hong, Y. K., Bruno, R. M. \& Sawtell, N. B. ${ }^{881}$ (2021), 'A rapid whisker-based decision underlying skilled locomotion in mice', Elife 10, e63596. 882

Wilson, M. A. \& McNaughton, B. L. (1993), 'Dynamics of the hippocampal ensemble code for ${ }_{883}$ space', Science 261(5124), 1055-1058.

Zaremba, J. D., Diamantopoulou, A., Danielson, N. B., Grosmark, A. D., Kaifosh, P. W., Bowler, 885 J. C., Liao, Z., Sparks, F. T., Gogos, J. A. \& Losonczy, A. (2017), 'Impaired hippocampal place 886 cell dynamics in a mouse model of the 22q11. 2 deletion', Nature neuroscience 20(11), 1612.

Zhao, X., Wang, Y., Spruston, N. \& Magee, J. C. (2020), 'Membrane potential dynamics underlying 888 context-dependent sensory responses in the hippocampus', Nature Neuroscience pp. 1-11. 889

Ziv, Y., Burns, L. D., Cocker, E. D., Hamel, E. O., Ghosh, K. K., Kitch, L. J., El Gamal, A. \& ${ }_{890}$ Schnitzer, M. J. (2013), 'Long-term dynamics of ca1 hippocampal place codes', Nature 891 neuroscience 16(3), 264. 


\section{Supplementary Materials}

- Figure S3; Simulation of BTSP-like vs continuously drifting place fields predict ${ }^{897}$ qualitatively different patterns of population tuning drift.

- Figure S4: Place fields do not continuously drift in either context.

- Figure S5: Experience-dependent correlations between field width and first-lap

- Figure S6: Interpretability of NMF solutions. 
A
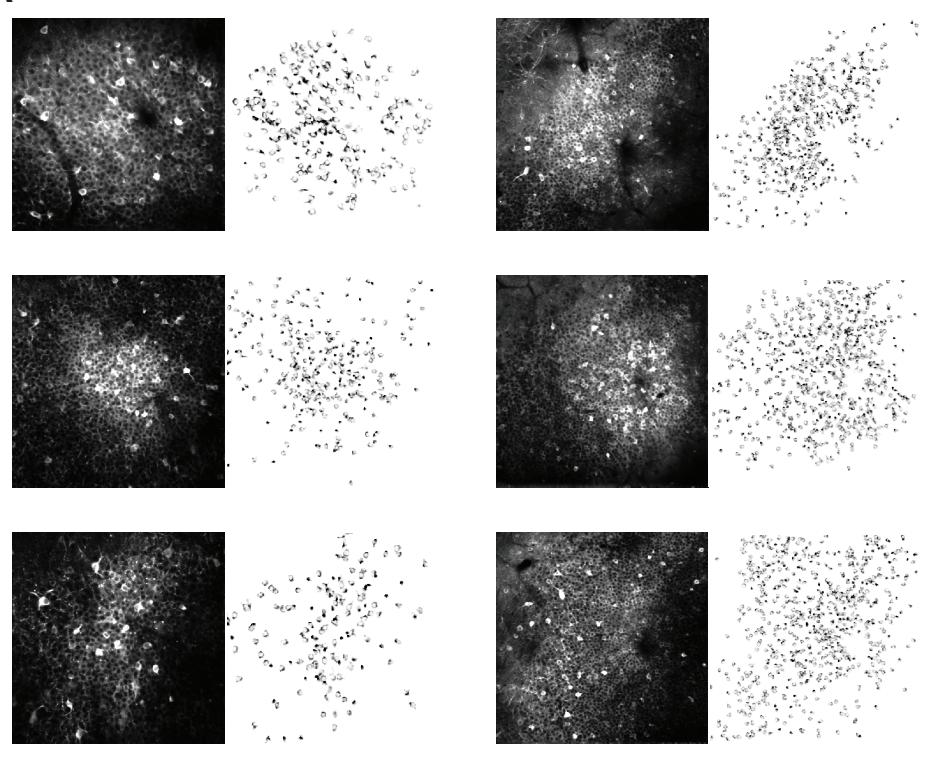

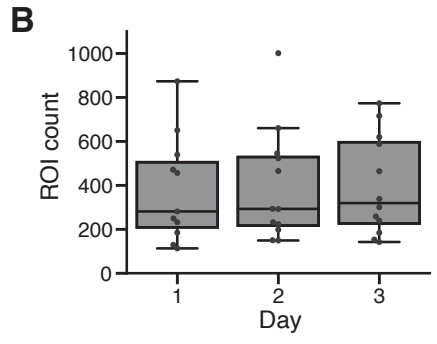

C

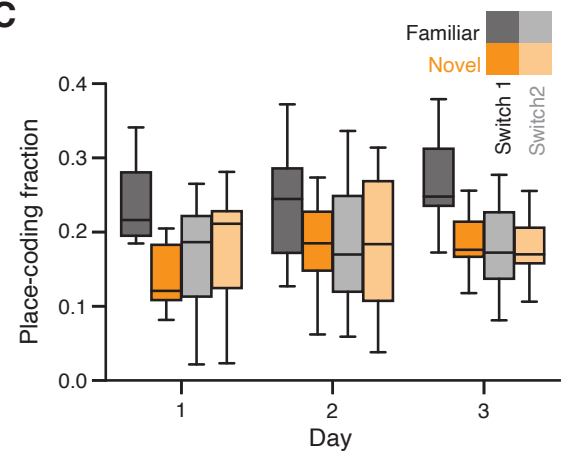

Figure S1. Data survey. A: Example FOVs and ROI masks for each mouse in the dataset. B: Number of ROIs detected during each experiment, by Day. C: Fraction of ROIs with a place field, by Day and trial block. 

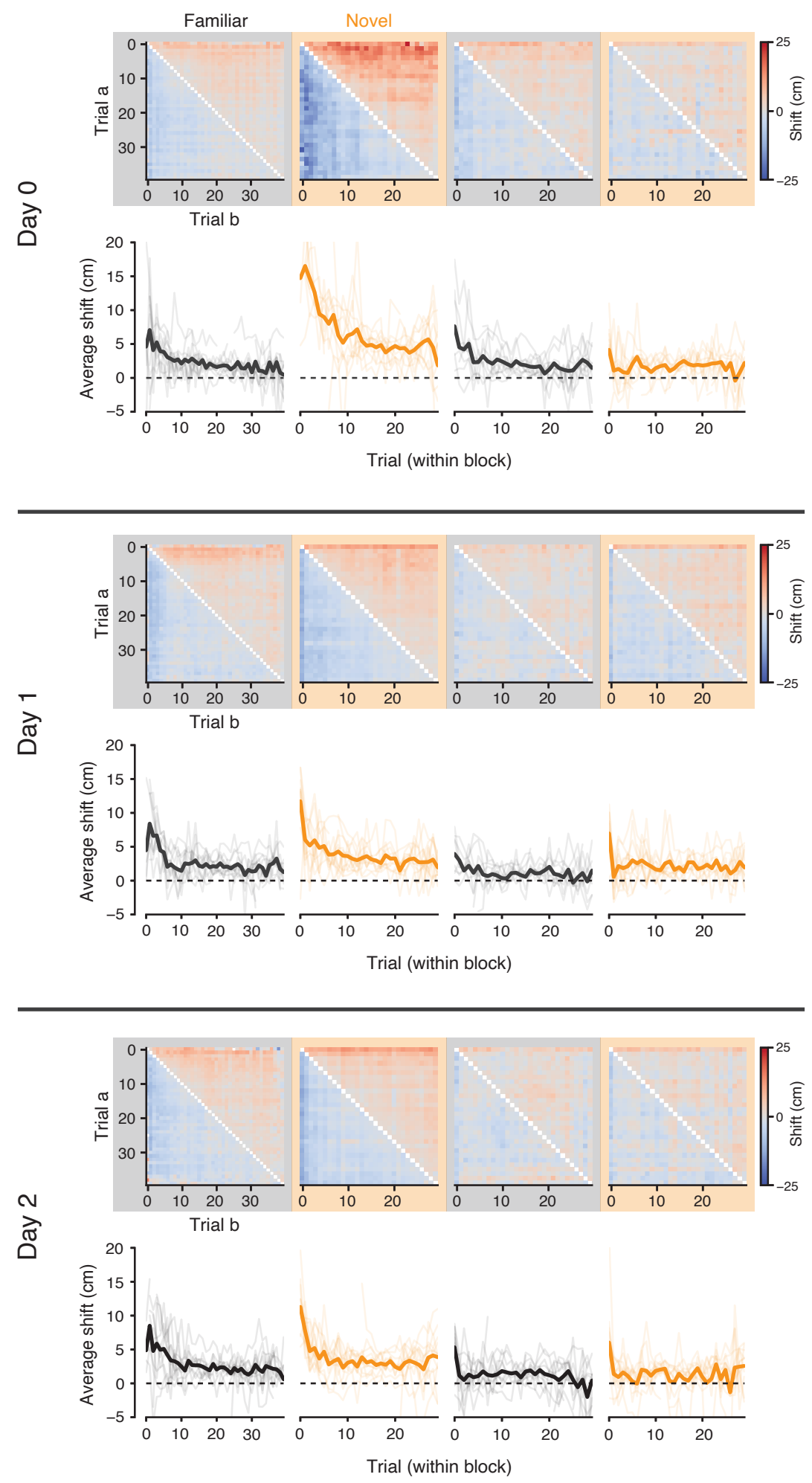

Figure S2. Population spatial drift is transient and experience-dependent. Population drift matrices and average trial shifts, as in Figure 2, shown for all three days of the experiment. The transient, backward shifting representation is specific to the novel context and strongest on the first day of the experiment. These shift distances are summarized and compared across days in Fig $2 \mathrm{E}, \mathrm{F}$. 
A

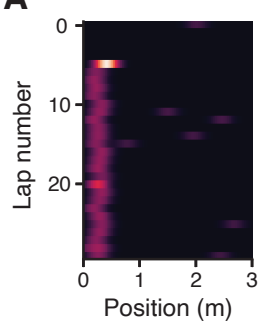

C
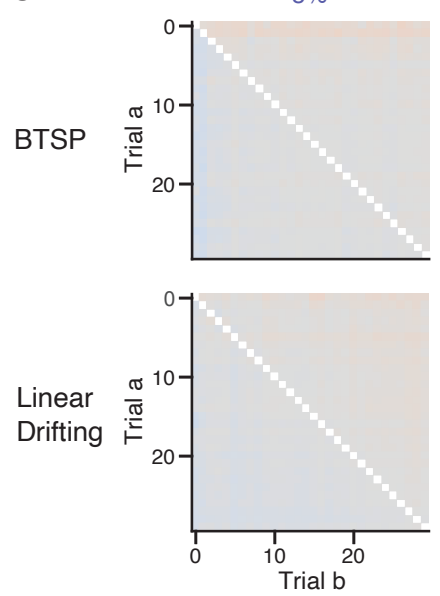

BTSP

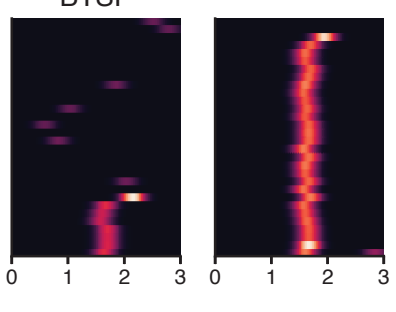

$30 \%$
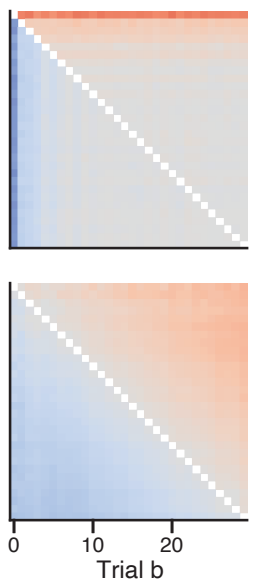

B

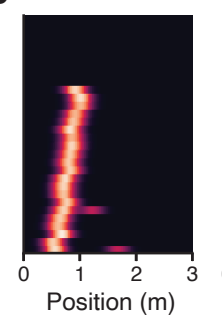

Linear Drifting
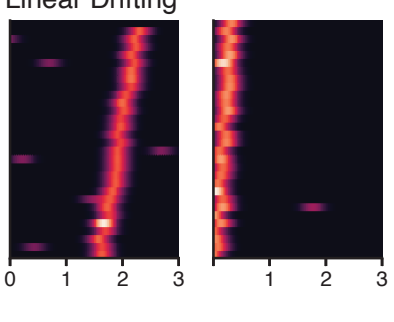

D

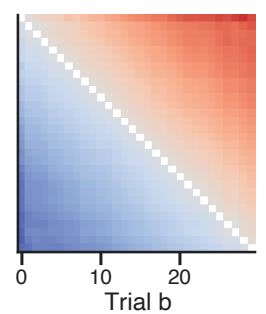

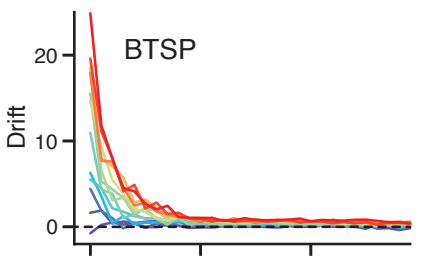
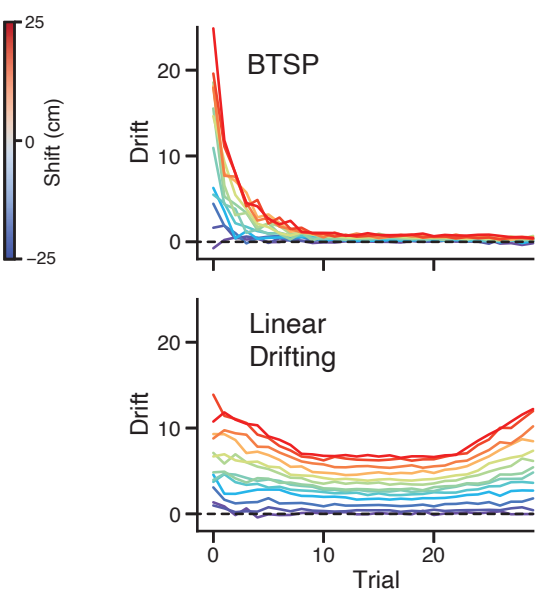

Figure S3. Simulation of BTSP-like vs continuously drifting place fields predict qualitatively different patterns of population tuning drift. A, B: Example simulated place fields under the two hypotheses. In both cases, the simulated populations acquired place fields over laps according to a geometric process. For BTSP simulations, each place field had a certain probability of exhibiting BTSP-like characteristics (forward-shifted and gain-modulated first lap activity). For drifting simulations, each place field had a certain probability of exhibiting a linear drift in its field location over laps. C: We studied how varying these characteristic probabilities affected the population tuning shift analysis shown in Fig. 2 In BTSP simulations, the population exhibited a transient backward drift concentrated on the first few laps in the session (i.e. when the majority of new place fields form), and this effect became exaggerated with greater BTSP probability. This situation closely resembles the data on the first exposure to the novel context during Day 1, consistent with the population drift arising due to the aggregate effect of many individual neurons undergoing a transient shift on their first active laps due to BTSP. In Linear Drifting simulations, population drift exhibits a very different pattern, where drift is largely a monotonic function of the temporal separation between trials (i.e., each diagonal of the shift matrix is mostly equal). D: Shift scores for each trial as in Fig. 2 shown for increasing probabilities of BTSP or linear drifting among the place cell population. Results in B and $\mathbf{C}$ are averages over 20 simulations, where each simulated population contained 300 neurons with a place-coding probability of $20 \%$. See Methods for additional simulation details. 


\section{A}
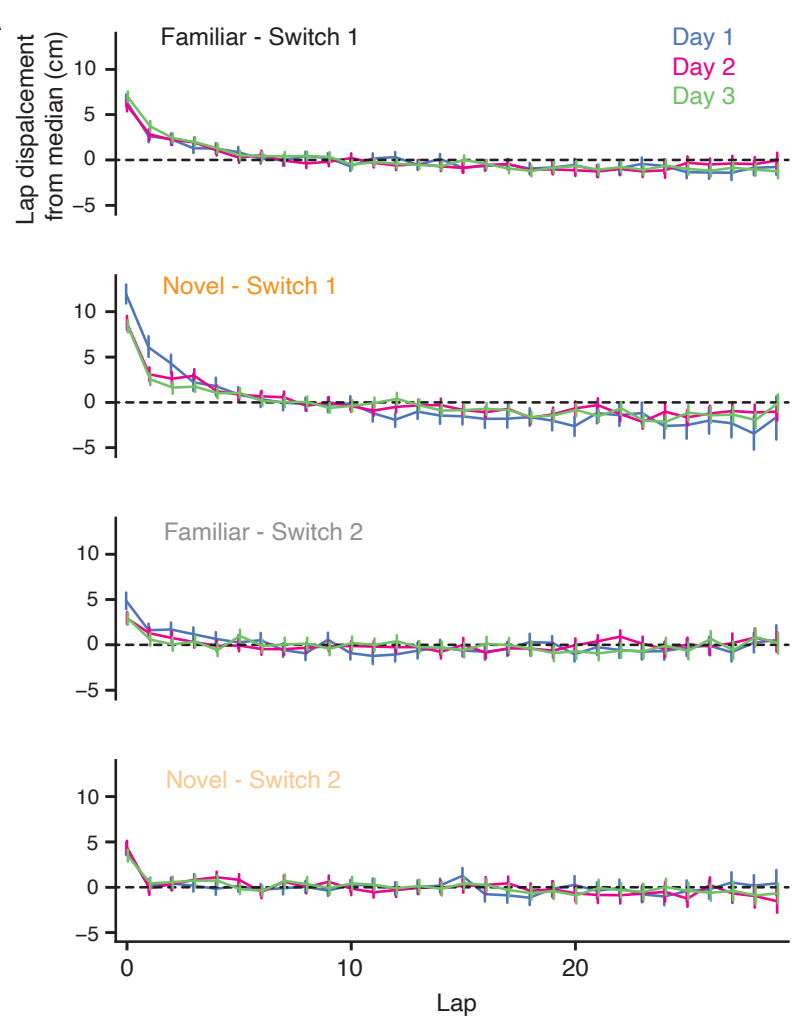
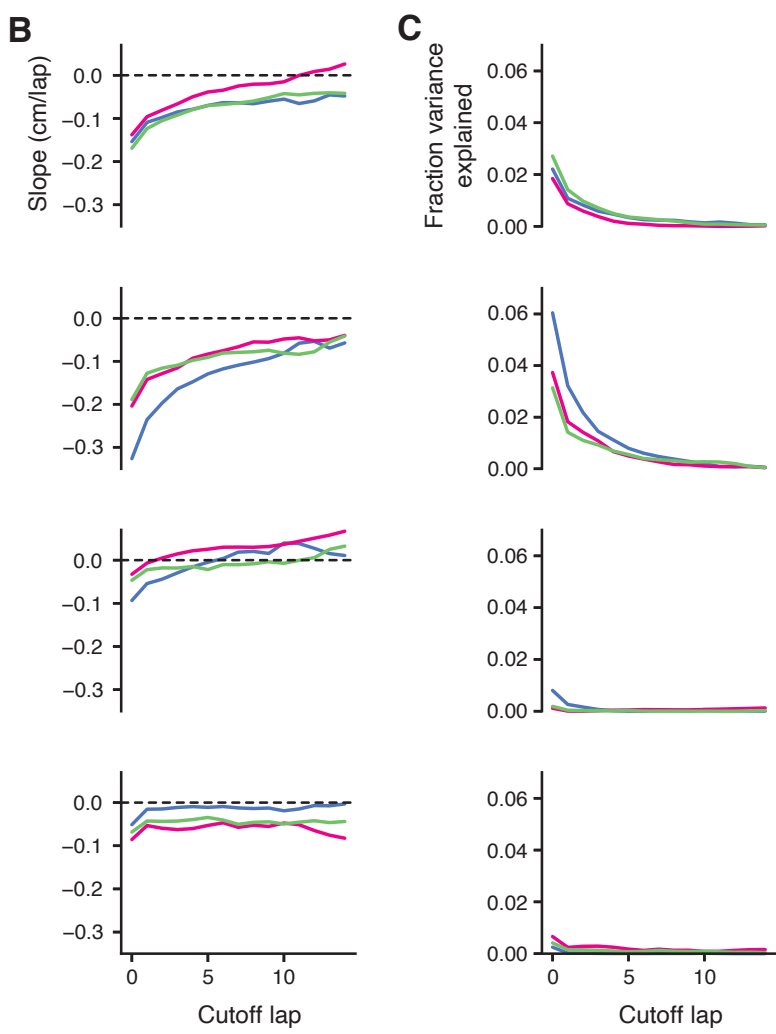
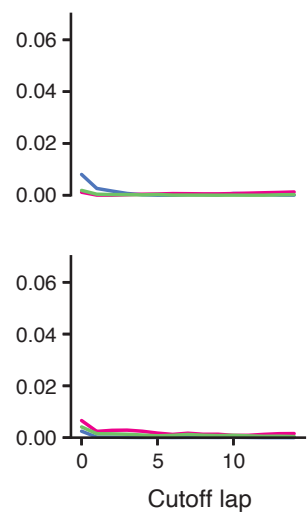

Figure S4. Place fields do not continuously drift in either context. A: Per-lap shifts in the center of mass (COM) of place field tuning, relative to the median lap COM. Place fields are grouped by context trial block and recording day. While there is a transient backward shift present across all conditions in the first few trials of the block, field drift quickly reaches a steady asymptote. B: Slope of the linear fit between lap number and field displacement in A. We recomputed the regression starting from different laps ('Cutoff lap'). When initial laps of the trial block are excluded, there linear fit suggests negligible field drift across all conditions $(<-0.1 \mathrm{~cm} / \mathrm{lap}$, which would accumulate to less than a single discrete spatial bin over the course of a context block). C: Fraction of variance explained by the linear fits described in $\mathbf{B}$. Excluding the transient drift at the beginning of the trial blocks, the linear trends generally explained $<1 \%$ of variance in place field displacements. 
A
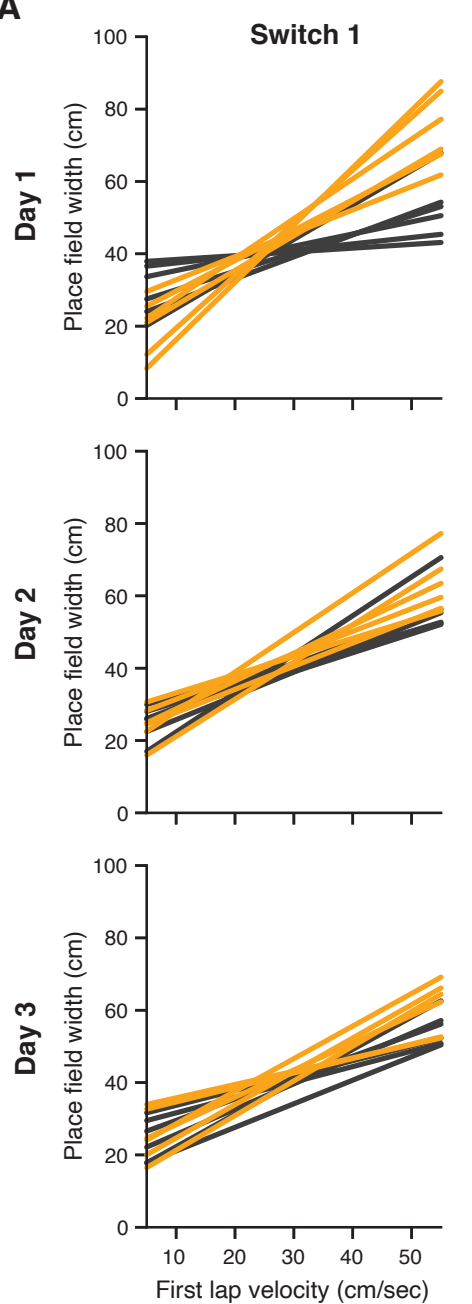

Switch 2
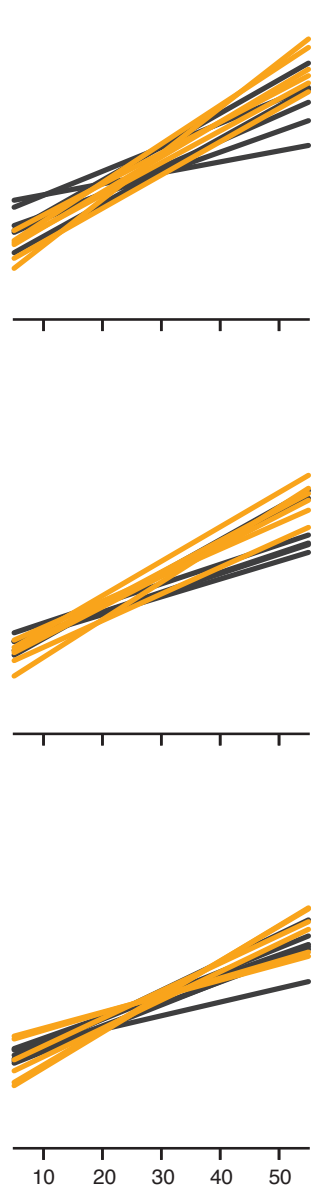

B

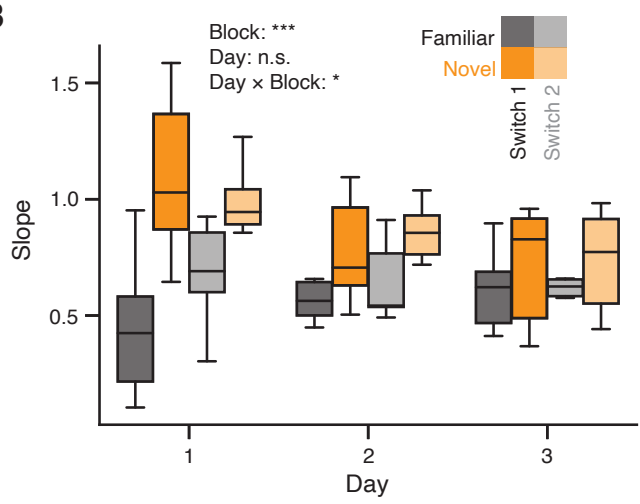

C

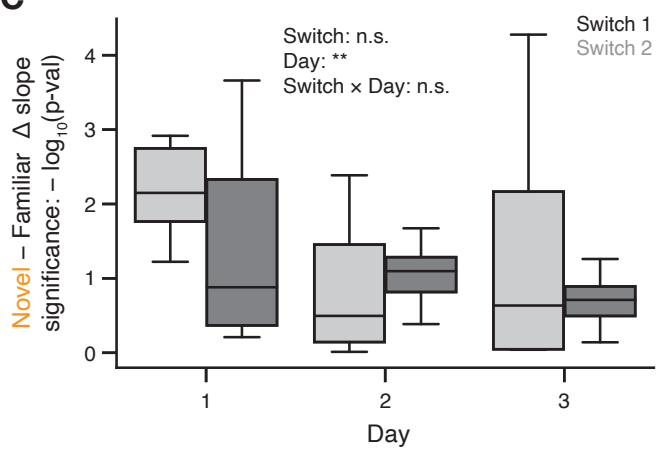

Figure S5. Experience-dependent correlations between field width and first-lap velocity are present in all mice. A: Linear fits for place field width as a function of the velocity of the animal during the first traversal of the place field, as in Fig $3 \mathrm{~F}$. Regression is computed separate for each mouse, plotted by switch (columns) and days (rows). B: Summary of regression slopes in A. The slope is greatest for the first exposure to the novel context on Day 1. Linear mixed effects model with main effects of Block and Day, significance inset. C: Summary of the significance of the difference between regression slopes in the Familiar vs Novel contexts, as in Fig $3 \mathrm{G}$, computed separately for each mouse, day, and switch. Linear mixed effects model with main effects of Switch and Day, significance inset. * $p<0.05,{ }^{* *} p<0.01,{ }^{* * *} p<0.001$ 
A

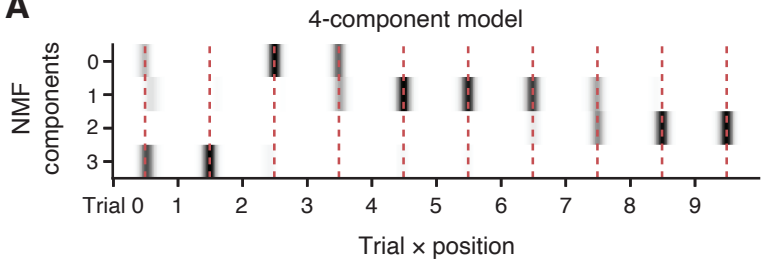

B

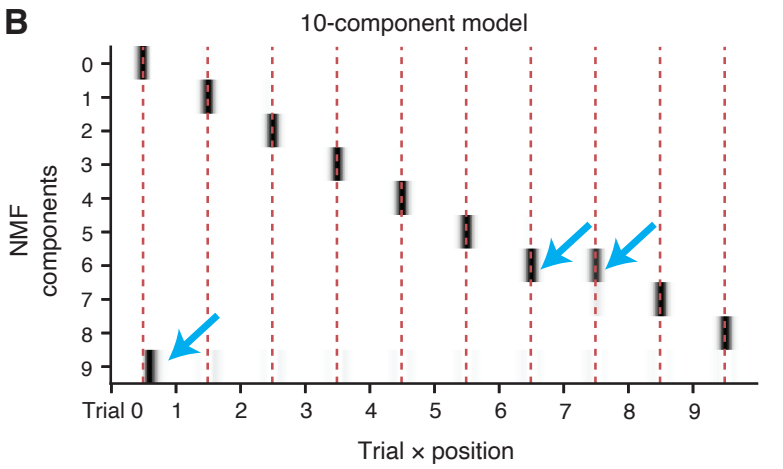

C

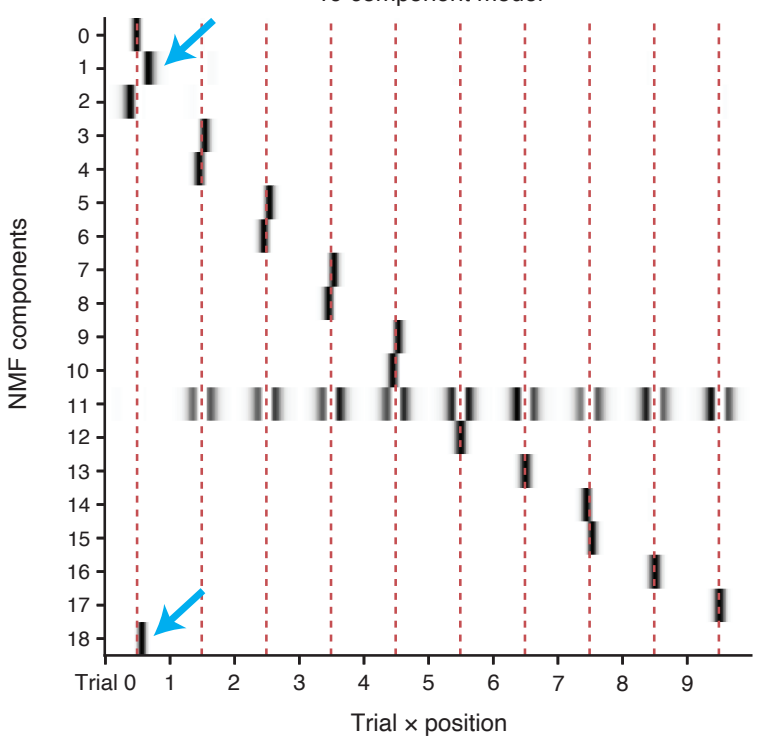

Figure S6. Interpretability of NMF solutions. Example NMF decompositions of the dataset of place fields for different number of components, to illustrate the deleterious behavior of the model for choices other than $n=11$. Red dashed lines indicate the average lap place field center. A: Here $n=4$ components. We found that when $n<10$, the components generally ended up reflecting mixtures of adjacent laps. This is likely because, if a place field is active in its field on lap $t$, it is arguably also likely to be active on laps $t-1$ and $t+1$, whereas its activity on more temporally distant laps is perhaps less certain. B: Here $n=10$ components. As $n$ increases, more laps obtain a dedicated component for modeling that lap's place field, but curiously when the number of components is equal to the number of laps, the model still prioritizes modeling the first-lap shift as a separate component. As a result, component 7 subsumes the place field activity of two laps. C: Here $n=19$ components. For all $n<11$, additional components tended to simply split the later lap place fields in half, symmetrically about the place field center (note how for the laps with two components, the component fields are narrower and lie on either side of the field center). Even still, the model places an outsized importance on capturing the first lap shift in greater detail, indicated by the accumulation of additional components right of the place field center. 
A

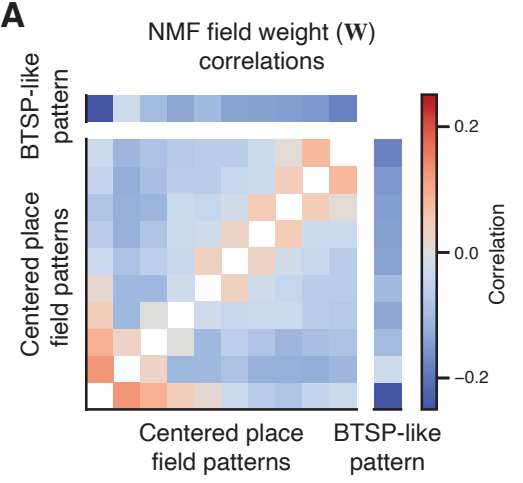

B

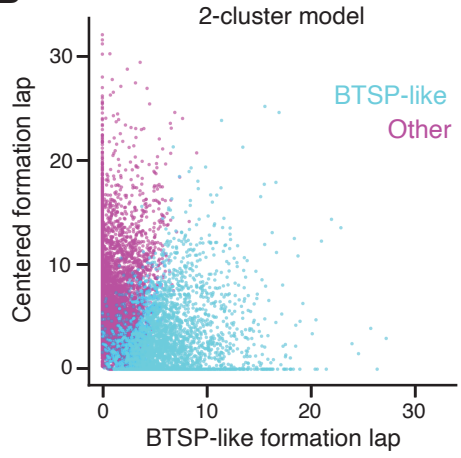

C

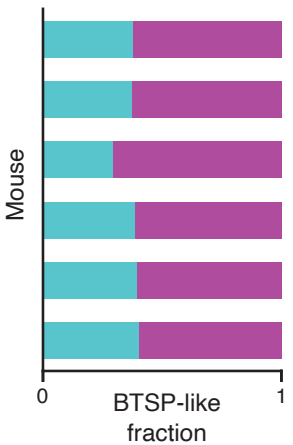

D

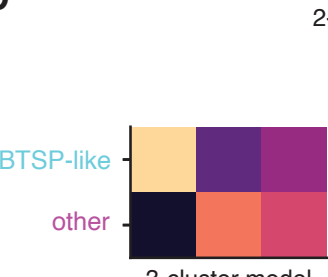

2-cluster vs higher order models

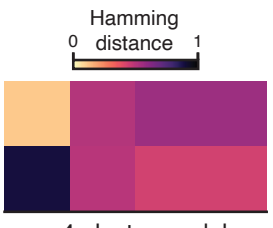

4-cluster model

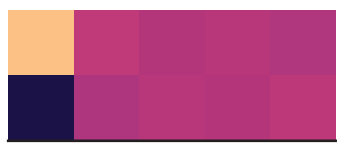

5-cluster model
E

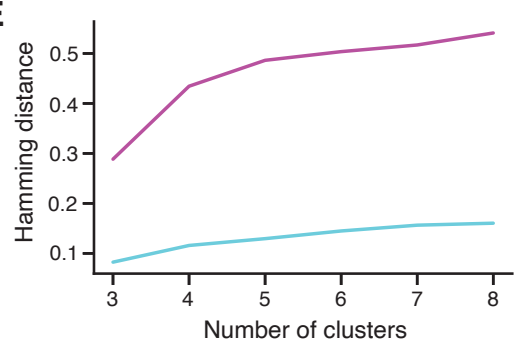

Figure S7. Isolating BTSP-like cells by clustering NMF pattern weights. A: Correlation between the place cell weights for each NMF pattern (i.e. correlations between the columns of W). The first 10 components are the centered place field patterns for each lap learned by the model, arranged sequentially. The last component is the shifted first lap pattern ("plateau" pattern). The first lap place field pattern and the plateau pattern show the strongest anti-correlation among all pattern pairs, suggesting that differential use of these patterns strongly clusters place fields, in line with the distribution shown in Fig $4 \mathrm{~F}$. The plateau component is also weakly anti-correlated with all other lap patterns, consistent with the idea that place fields with a plateau on their first lap will generally have higher amplitude first lap responses compared to later laps. Fields that highly weight the plateau component would then tend to have lower weights for later laps, while fields that down-weight the plateau component will have higher weights for later laps (since each field is mean-normalized across laps). B: We applied K-means clustering to place fields in the 11-dimensional NMF space (i.e. clustering the rows of $\mathbf{W}$ ) to identify the BTSP-like group of place fields analyzed in Fig. 5. We set $K=2$ and found that this was sufficient to reliably uncover a "BTSP-like" group of place fields. Here we show the distribution of place fields in the plane of the centered and shifted first lap patterns, colored by cluster. The BTSP-like cluster subsumes virtually all place fields with weight on the shifted "plateau" pattern. C: A similar fraction of place fields are identified as BTSP-like for each mouse in the dataset. D: We compared the cluster labels in the 2-cluster model used in the main figures to higher order models. For each order $n$ model, we computed the normalized hamming distance between its $n$ clusters and the original 2 clusters (BTSP-like/other). A distance of 0 indicates a pair of cluster labelings is identical, whereas 1 indicates they are inverted. Higher order models always find a very similar cluster to the original BTSP-like group, and tend to divide the original "other" group into smaller partitions to populate the additional clusters. E: Summary of original BTSP/other cluster survival as the number of clusters is increased. For the BTSP-like and other clusters, we identified the new cluster in each higher order model with the lowest hamming distance (i.e., most similar). The BTSP-like cluster is highly stable. 


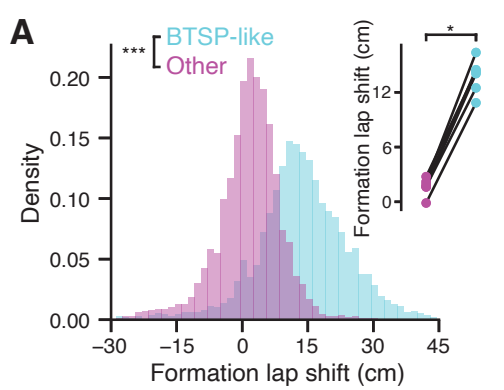

D

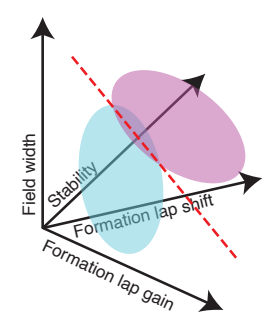

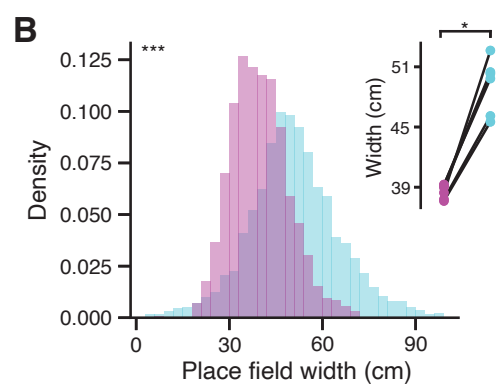

E

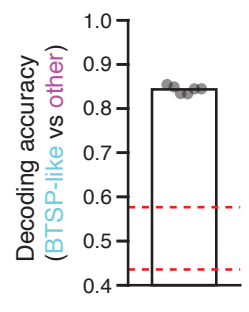

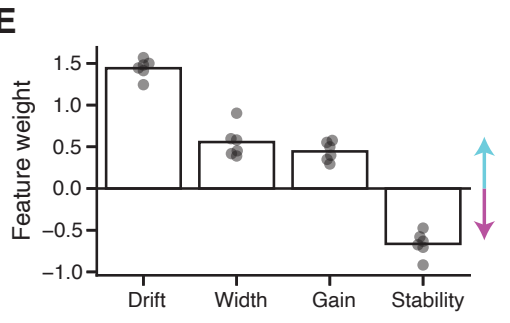

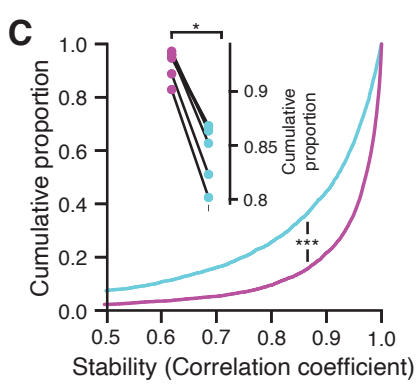

$\mathbf{F}$

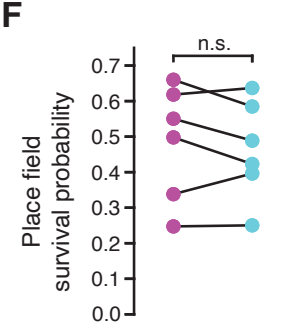

Figure S8. Characteristics of BTSP-like place fields. A: Spatial shift between activity on the first lap of place field activity and the activity on remaining laps, for BTSP-like and other place fields. Left: shift distributions for the dataset. Right: average first lap shift for each group, by mouse. B: Width of place fields, excluding first lap activity. Plotted as in A. C: Stability of place fields, computed as the correlation between spatial tuning on even vs odd laps (excluding the first lap). Plotted as in A. D: Decoding BTSP-like vs Other labels from field characteristics. Left: schematic of the analysis. Each place field was represented as a point in a 4-dimensional space, described by its first lap field gain (Fig. 5), first lap shift, place field width, and stability. A linear SVM was then used to classify fields as either BTSP-like or Other in this space. Right: results of decoding analysis. Plotted is the average cross-validated decoding performance for each mouse. Chance level is demarcated in red, obtained by constructing a null distribution through shuffling place field labels. See Methods for details on cross-validation and significance testing. E: Coefficients learned for each feature in the decoder (averaged across cross-validation folds). F: Place field survival probability. For each place field identified in the first block of either context, we asked whether we also detected a place field at that location in the second block of the same context during that experiment. Survival probabilities are computed by mouse. 
A

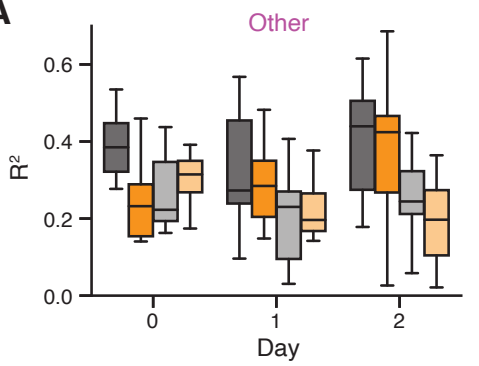

BTSP-like

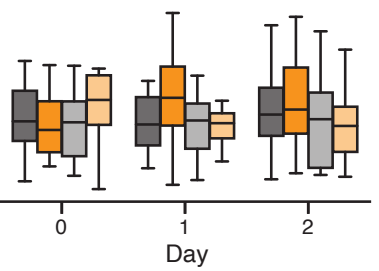

B

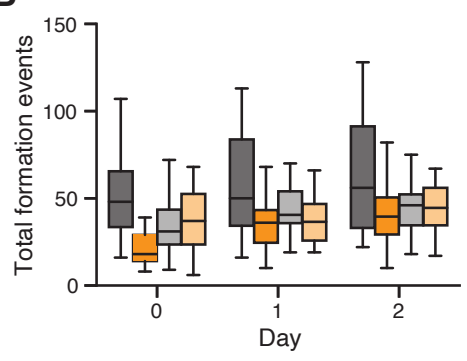

Familiar
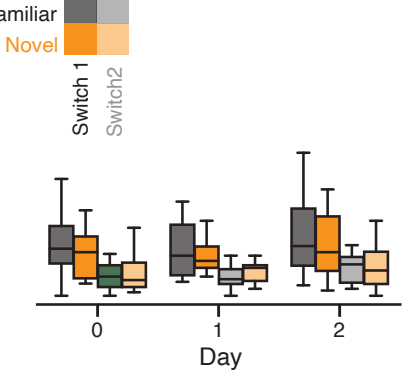

\section{C}

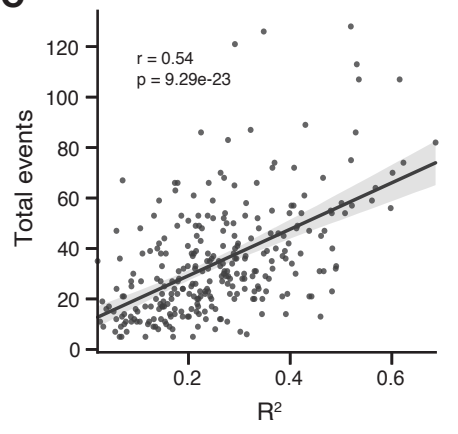

Figure S9. Effect of velocity on the spatial distribution of BTSP across experience. A: As in Fig. 6 , we fit a linear model that predicted the number of place field formation events at each location as a function of the filtered velocity of the animal. Here we do this analysis separately for each experiment and context block, to identify any changes in the fit quality over experience. Results are plotted separately for 'BTSP-like' and 'Other' place fields. The quality of the fit varies more for 'Other'. B: Total number of place fields that formed in each of the conditions plotted in A. C: Correlation between place field counts and the fit quality of the velocity-field density model. Variability in model fit quality is largely accounted for by differences in the number of place field formation events observed during those conditions. Intuitively, if fewer events are sampled in a given condition, it is harder to estimate the true spatial distribution of place field formation. 